\title{
A fast-marching like algorithm for Geometrical Shock Dynamics
}

\author{
Y. Noumir ${ }^{\mathrm{a}}$, A. Le Guilcher ${ }^{\mathrm{b}}$, N. Lardjane ${ }^{\mathrm{c}, *}$, R. Monneau ${ }^{\mathrm{b}}$ \\ ${ }^{a}$ LRC-MESO, CMLA, ENS Cachan, 61 avenue du Président Wilson 92235 Cachan, \\ France \\ ${ }^{b}$ CERMICS-ENPC, Cité Descartes, 6-8 Avenue Blaise Pascal, Champs-sur-Marne, \\ 77455 Marne-la-Vallée Cedex 2, France \\ ${ }^{c} C E A, D A M, D I F$, F-91297 Arpajon, France
}

\begin{abstract}
We develop a new algorithm for the computation of the geometrical shock dynamics model (GSD). The method relies on the fast-marching paradigm and enables the discrete evaluation of the first arrival time of a shock wave and its local velocity on a cartesian grid. The proposed algorithm is based on a second order upwind finite difference scheme and reduces to a local nonlinear system of two equations solved by an iterative procedure. Reference solutions are built for a smooth radial configuration and for the 2D Riemann problem. The link between the GSD model and p-systems is given. Numerical experiments demonstrate the accuracy and the ability of the scheme to handle singularities.
\end{abstract}

Keywords: geometrical shock dynamics, fast-marching method, level-set method, Riemann problem, finite difference method, shock wave, p-system

\section{Introduction}

In 1957, when G.B. Whitham published the Geometrical Shock Dynamics (GSD) model [28], he qualified it as a relatively simple approximate method developed for treating problems of the diffraction and stability of shock waves. The simplicity comes from the fact that the shock front is seen as a surface

\footnotetext{
*Correponding author. Tel.: +33 169264000

Email address: nicolas.lardjane@cea.fr (N. Lardjane)
} 
evolving under its own local speed and curvature, independently of the postshock flow. The shock adjusts itself to changes in the geometry only [29]. As explained by Best [8], Whitham considered the motion of a shock into a uniform gas at rest, down a tube of slowly varying cross sectional area, $A$, and under some physically grounded hypothesis, he obtained an expression relating the local shock Mach number, $M$, to $A$, now known as the A-M relation [31] (see also (9) and (4)). The GSD model reads

$$
M(\mathbf{x})|\nabla \alpha(\mathbf{x})|=1, \quad \operatorname{div}\left(\frac{\mathbf{n}(\mathbf{x})}{\mathrm{A}(\mathrm{M}(\mathbf{x}))}\right)=0
$$

where $\alpha$ gives the shock position [29] and $\mathbf{n}=\frac{\nabla \alpha}{|\nabla \alpha|}$ is the local normal to the front. This model is hyperbolic provided that $A^{\prime}(M)<0$, and can thus develop disturbances on the front which are the trace of waves, not modeled, behind the shock. In practice, GSD has proven to be fairly accurate for diffraction around a corner, non-regular Mach reflection [29], or accelerating shocks and shown only little deviation for expanding decelerating flows [5]. Whitham's model has been extended to take into account unsteady flow behind the shock $[8,9,10]$, non-uniform gases properties [20], and has been applied, among others, to imploding shock waves [11, 1], atmospheric propagation [7], detonation in explosives [2, 3, 6], supersonic engine unstart [27] and astrophysics [14].

This success, linked to the compact model formulation and the dimensional reduction, was supported by the development of three kinds of algorithms. (i) Lagrangian, or front-tracking, methods have first been experimented $[15,18]$. In such an approach, the shock front is explicitly discretized by markers evolved in time and regularly resampled. This method is natural and quite accurate but difficult to implement in three dimensions, mainly when surface merging or breaking is expected. (ii) Eulerian conservative algorithms $[19,20]$ reduce this difficulty but do not take any advantage of the front locality. Furthermore, they rely on the definition of an a priori propagation direction, not always easy to determine. (iii) Localized level-set methods are a good compromise since they handle any kind of surface deformation but in an implicit way. The front shock is obtained from a table of arrival time, also called burn table. A 3D unsteady algorithm, based on the Hamilton-Jacobi form of the GSD system (1) [17], is described in [2, 3, 4] for Detonation Shock Dynamics. It compares well with reactive Eulerian model results at a much lower CPU time. Nevertheless, due to the nonlinear nature 
of GSD equations, unphysical shocks can form away from the front position and a frequent resampling of the signed distance is mandatory [23].

In this article we propose an alternative approach based on the levelset fast-marching paradigm [22], which combines the flexibility of (iii) and the locality of (i) while remaining easy to implement. The first and second ordrer specific algorithms are decribed in section 2. Reference solutions for a smooth radial problem and the GSD Riemann problem are derived in section 3. In section 4 , the comparison to numerical results indicates that a second order scheme is mandatory for non smooth problems. At last, conclusions are summarized in section 5 .

\section{A fast-marching like GSD scheme}

In 1988 Osher and Sethian [17] introduced the Eulerian level-set method to solve Hamilton-Jacobi equations and the eikonal equation in particular. Unlike the Lagrangian approach, the level-set method is simple to implement in 3D, high-order extensions are readily derived and topological properties of the front, as the curvature, are easily calculated. However, the level-set mehod, of complexity $O\left(N^{3}\right)$, can be quite time consuming when the number of grid point, $N$, is large. In the past two decades, several improvements have been proposed in order to reduce this complexity and at same time to enhance the accuracy. Among them, the most popular approach is the FastMarching Method (FMM) developed by Sethian [22] and used with success in a large variety of applications. Assuming a single pass front, the complexity of the algorithm reduces to $O(N \log N)$ and even to $O(N)$ under some further assumptions [30].

In this section, we introduce a second-order method to solve the GSD model (1), on a cartesian grid, based on the fast-marching paradigm. We first reformulate the model as a coupled eikonal-transport system to facilitate its discretisation. The numerical method, boundary treatment and implementation details are then given.

\subsection{A modified transport equation}

As in the work of Besset [7] or Aslam [2], the GSD model (1) is rewritten under the local form 


$$
\left\{\begin{array}{c}
M|\nabla \alpha|=1 \\
M \frac{\nabla \alpha}{|\nabla \alpha|} \cdot \nabla M=\dot{M}(M, \kappa)
\end{array}\right.
$$

with the initial boundary conditions

$$
\left\{\begin{array}{l}
\alpha_{\Gamma_{\Gamma_{0}}}=\alpha_{0}, \\
M_{\left.\right|_{\Gamma_{0}}}=M_{0},
\end{array}\right.
$$

where $\alpha_{0}$ and $M_{0}$ are given functions on the hypersurface $\Gamma_{0}$, the shock initial position. The GSD closure, linking the local Mach number, $M$, and the mean curvature of the front, $\kappa=\operatorname{div}(\mathbf{n})$, reads

$$
\dot{M}(M, \kappa)=-\frac{M^{2}-1}{\lambda(M)} \kappa,
$$

where $M \geq 1$ and

$$
\left\{\begin{array}{l}
0<\lambda(M)=\left(1+\frac{2}{\gamma+1} \frac{1-\mu^{2}}{\mu}\right)\left(1+2 \mu+\frac{1}{M^{2}}\right), \\
1 \geq \mu=\sqrt{\frac{(\gamma-1) M^{2}+2}{2 \gamma M^{2}-(\gamma-1)}}
\end{array}\right.
$$

$\gamma>1$ being the gas polytropic coefficient, see [29] for details.

In practice, the discretization of the mean curvature is difficult, due to the mixed derivatives of $\alpha$, especially in the context of the fast-marching method when neighboring points are not yet assigned a value. For this reason we choose to reformulate the transport equation on the Mach number as a convection-diffusion one.

By combining the eikonal equation, $M|\nabla \alpha|=1$, and the normal definition, $\mathbf{n}=\nabla \alpha /|\nabla \alpha|$, one checks that the mean curvature of the front reads $\kappa=\nabla M \cdot \nabla \alpha+M \Delta \alpha$. Transport equation (2b) is then reformulated as

$$
\nabla M \cdot \nabla \alpha=\mathcal{S}(M) \Delta \alpha
$$


where $\mathcal{S}(M)=-\frac{M\left(M^{2}-1\right)}{M^{2}(\lambda(M)+1)-1}$ is nonpositive and smooth and for $M>1$. The reformulated boundary value problem to be solved is then

$$
\left\{\begin{array}{c}
M|\nabla \alpha|=1 \\
\nabla M \cdot \nabla \alpha=\mathcal{S}(M) \Delta \alpha
\end{array}\right.
$$

where the source term is now easier to discretize.

Note that this system is not in a conservative form, which could raise difficulties to handle front discontinuities, but as we shall see in section 4.3 the numerical scheme performs well in practice.

\subsection{Level-set method for the eikonal equation}

The level-set function $\alpha$ is solution of the eikonal equation (5a) on $\Omega$ together with the initial condition (3a) on $\Gamma_{0}$. We solve it on a uniform cartesian mesh of the domain $\Omega \equiv[0, L x] \times[0, L y] \times[0, L z] \subset \mathbb{R}^{3}$ with grid spacings $\triangle x, \triangle y$ and $\triangle z$. Let $\alpha_{i, j, k}$ and $M_{i, j, k}$ be the approximate solution at a grid point, i.e. $\alpha_{i, j, k}=\alpha\left(x_{i}, y_{j}, z_{k}\right)$ and $M_{i, j, k}=M\left(x_{i}, y_{j}, z_{k}\right)$ where $x_{i}=i \triangle x, y_{j}=j \triangle y$ and $z_{k}=k \triangle z$. We denote by $u_{l}$, $v_{l}$ et $w_{l}$ (resp. $u_{r}, v_{r}$ et $w_{r}$ ) the backward (resp. forward) approximation of the derivatives of $\alpha$ at $\left(x_{i}, y_{j}, z_{k}\right)$ along $x, y$ and $z$ respectively.

The discrete form of $(5 \mathrm{a})$ reads

$$
M_{i, j, k} \hat{H}\left(u_{l}, u_{r}, v_{l}, v_{r}, w_{l}, w_{r}\right)=1,
$$

where $\hat{H}$ is chosen as the numerical Hamiltonian of Godunov [25]:

$$
\hat{H}\left(u_{l}, u_{r}, v_{l}, v_{r}, w_{l}, w_{r}\right)=\sqrt{\max ^{2}\left(u_{l}^{+}, u_{r}^{-}\right)+\max ^{2}\left(v_{l}^{+}, v_{r}^{-}\right)+\max ^{2}\left(w_{l}^{+}, w_{r}^{-}\right)},
$$

with the notations: $x^{+}=\max (x, 0)$ and $x^{-}=\max (-x, 0)$ for $x \in \mathbb{R}$. This upwind scheme has been sucessfully used in several applications ( $c f$. [16], [22]). It has the ability to capture viscosity solutions of the eikonal equation [12] and do not smear out sharp discontinuities excessively [25], which are desirable features in the case of GSD where front singularities may appear. 
At first order, the discrete derivatives of $\alpha$ are written:

$$
\begin{aligned}
& u_{l}=\left(D_{x}^{-} \alpha\right)_{i, j, k}:=\frac{\alpha_{i, j, k}-\alpha_{i-1, j, k}}{\triangle x} \mid u_{r}=\left(D_{x}^{+} \alpha\right)_{i, j, k}:=\frac{\alpha_{i+1, j, k}-\alpha_{i, j, k}}{\triangle x} \\
& v_{l}=\left(D_{y}^{-} \alpha\right)_{i, j, k}:=\frac{\alpha_{i, j, k}-\alpha_{i, j-1, k}}{\triangle y} \quad v_{r}=\left(D_{y}^{+} \alpha\right)_{i, j, k}:=\frac{\alpha_{i, j+1, k}-\alpha_{i, j, k}}{\triangle y} . \\
& w_{l}=\left(D_{z}^{-} \alpha\right)_{i, j, k}:=\frac{\alpha_{i, j, k}-\alpha_{i, j, k-1}}{\triangle z} \mid w_{r}=\left(D_{z}^{+} \alpha\right)_{i, j, k}:=\frac{\alpha_{i, j, k+1}-\alpha_{i, j, k}}{\triangle z}
\end{aligned}
$$

The extension to arbitary higher orders is possible, we restrict ourselves to second-order in this work.

At second order, the discrete derivatives of $\alpha$ are written:

$$
\begin{aligned}
& u_{l}=\left(D_{x}^{-} \alpha\right)_{i, j, k}+\frac{\triangle x}{2}\left(D_{x}^{-}\left(D_{x}^{-} \alpha\right)\right)_{i, j, k} \mid u_{r}=\left(D_{x}^{+} \alpha\right)_{i, j, k}-\frac{\triangle x}{2}\left(D_{x}^{+}\left(D_{x}^{+} \alpha\right)\right)_{i, j, k} \\
& v_{l}=\left(D_{y}^{-} \alpha\right)_{i, j, k}+\frac{\triangle y}{2}\left(D_{y}^{-}\left(D_{y}^{-} \alpha\right)\right)_{i, j, k} \mid \begin{array}{l}
v_{r}=\left(D_{y}^{+} \alpha\right)_{i, j, k}-\frac{\triangle y}{2}\left(D_{y}^{+}\left(D_{y}^{+} \alpha\right)\right)_{i, j, k} \\
w_{l}=\left(D_{z}^{-} \alpha\right)_{i, j, k}+\frac{\triangle z}{2}\left(D_{z}^{-}\left(D_{z}^{-} \alpha\right)\right)_{i, j, k} \mid w_{r}=\left(D_{z}^{+} \alpha\right)_{i, j, k}-\frac{\triangle z}{2}\left(D_{z}^{+}\left(D_{z}^{+} \alpha\right)\right)_{i, j, k}
\end{array} .
\end{aligned}
$$

We point out that there is no limiting function. In the context of the FMM, a switching mechanism is rather used as we shall see later.

\subsection{The standard fast-marching method}

Following Sethian [22], the classical FMM is now outlined. In this method, the velocity of propagation is assumed to be known and of constant sign. The front is thus "single pass" and one needs to calculate the value of $\alpha$ only in the vicinity of it. The CPU time is then dramatically reduced and, interrelated, the monotonicity of the scheme is guaranted by following the direction in which the information flows. This is done by propagating the solution from lower to higher values of the level-set function $\alpha$. To this end, the grid points are partitioned into three groups, namely:

(1) Known is the set of vertices where the values of $\alpha$ are known, i.e. the vertices already intercepted by the front;

(2) NarrowBand is the set of all neighboring vertices of Known, i.e. the vertices that are about to be intercepted by the front; 
(3) Far is the set of vertices that are neither in Known nor in NarrowBand. In the Far set, $\alpha$ is assigned a huge initial value INF.

Think of the NarrowBand set as a buffer zone that serves to start the calculation from the vertices of Known and to spread the information to the vertices of the Far set. For every element of the NarrowBand, test values of $\alpha$ are calculated from the points of the Known set only, and then the point of the NarrowBand corresponding to the minimal test value is validated. This means that the value of $\alpha$ at this point is now defined as the minimal test value. A second step consists in including this point in the Known set and adding its close Far neighbors as new points of the NarrowBand. By repeating the previous steps, as long as the NarrowBand is not-empty, the level-set function is calculated in the whole computational domain. The starting values of this process are set by the initial conditions on $\Gamma_{0}$. It is worth mentioning that the most time consuming step in this algorithm is the search of the point with the minimal test value and thus the performance of the FMM depends on it.

At a point $(i, j, k)$ of interest of the NarrowBand, we can deduce from (6) that the test value $\vartheta$ of $\alpha$ is calculated by solving the following quadratic equation:

$$
\sum_{\nu=1}^{3} \max ^{2}\left(\frac{\vartheta-t_{i, j, k}^{\nu^{-}}}{\triangle_{\nu}^{-}}, \frac{\vartheta-t_{i, j, k}^{\nu^{+}}}{\triangle_{\nu}^{+}}, 0\right)=\frac{1}{M_{i, j, k}^{2}},
$$

with the notations

$\triangle_{1}^{ \pm}=\triangle x\left(1-\frac{s_{i \pm 1, j, k}^{ \pm x}}{3}\right), \triangle_{2}^{ \pm}=\triangle y\left(1-\frac{s_{i, j \pm 1, k}^{ \pm y}}{3}\right), \triangle_{3}^{ \pm}=\triangle z\left(1-\frac{s_{i, j, k \pm 1}^{ \pm z}}{3}\right)$,

and with the following generic switches

$$
\begin{aligned}
& s_{i, j, k}^{ \pm x}=\left\{\begin{array}{ll}
1 & \text { if } \alpha_{i \pm 1, j, k}<\alpha_{i, j, k} \\
0 & \text { otherwise }
\end{array} s_{i, j, k}^{ \pm y}= \begin{cases}1 & \text { if } \alpha_{i, j \pm 1, k}<\alpha_{i, j, k} \\
0 & \text { otherwise }\end{cases} \right. \\
& s_{i, j, k}^{ \pm z}= \begin{cases}1 & \text { if } \alpha_{i, j, k \pm 1}<\alpha_{i, j, k} \\
0 & \text { otherwise }\end{cases}
\end{aligned}
$$


where the coefficients $\left(t_{i, j, k}^{\nu^{ \pm}}\right)_{1 \leq \nu \leq 3}$ are given by:

$$
\mid \begin{gathered}
t_{i, j, k}^{1^{ \pm}}=\alpha_{i \pm 1, j, k}+\frac{s_{i \pm 1, j, k}^{ \pm x}}{3}\left(\alpha_{i \pm 1, j, k}-\alpha_{i \pm 2, j, k}\right) \\
t_{i, j, k}^{2^{ \pm}}=\alpha_{i, j \pm 1, k}+\frac{s_{i, j \pm 1, k}^{ \pm y}}{3}\left(\alpha_{i, j \pm 1, k}-\alpha_{i, j \pm 2, k}\right) \\
t_{i, j, k}^{3^{ \pm}}=\alpha_{i, j, k \pm 1}+\frac{s_{i, j, k \pm 1}^{ \pm z}}{3}\left(\alpha_{i, j, k \pm 1}-\alpha_{i, j, k \pm 2}\right)
\end{gathered}
$$

The switches take into account the local direction of propagation. They ensure the causality condition by setting a zero value when the information at the second order is not available in that direction.

Remark 2.1. At first-order of accuracy, one can take all switches equal to zero and one has $\triangle_{1}^{ \pm}=\triangle x, \triangle_{2}^{ \pm}=\triangle y, \triangle_{3}^{ \pm}=\triangle z$, and $t_{i, j, k}^{1^{ \pm}}=\alpha_{i \pm 1, j, k}$, $t_{i, j, k}^{2^{ \pm}}=\alpha_{i, j \pm 1, k}, t_{i, j, k}^{3^{ \pm}}=\alpha_{i, j, k \pm 1}$.

\subsection{Full discretisation of the GSD system}

Following the fast-marching paradigm, a method for solving the coupled system of equations (5) is now explained. Since $M$ is an unknown of the problem, one has to calculate test values of $\alpha$ (i.e. $\vartheta$ ) and of $M$ (i.e. $m$ ), at the same time, for each point of the NarrowBand, leading to a local non linear system in $\vartheta$ and $m$. The discrete velocity $M_{i, j, k}$ is now replaced by $m$ in the equation (7) and the discretization of the transport equation (5b) is done as follows.

The advection part of equation (5b) reads $\nabla M \cdot \nabla \alpha$. Following the upwind direction of discretization for $\nabla \alpha$ ensures that only receivable points are used in the computation of $\nabla M$. More precisely, we have:

$$
\left.(\nabla M \cdot \nabla \alpha)\right|_{i, j, k}=\sum_{\nu=1}^{3}\left[\left(\frac{\vartheta-t_{i, j, k}^{\nu^{-}}}{\triangle_{\nu}^{-}}\right)^{+} \frac{m-\ell_{i, j, k}^{\nu^{-}}}{\triangle_{\nu}^{-}}-\left(\frac{t_{i, j, k}^{\nu^{+}}-\vartheta}{\triangle_{\nu}^{+}}\right)^{-} \frac{\ell_{i, j, k}^{\nu^{+}}-m}{\triangle_{\nu}^{+}}\right]
$$


where the coefficients $\left(\ell_{i, j, k}^{\nu^{ \pm}}\right)_{1 \leq \nu \leq 3}$ take the form:

$$
\mid \begin{aligned}
& \ell_{i, j, k}^{1^{ \pm}}=M_{i \pm 1, j, k}+\frac{s_{i \pm 1, j, k}^{ \pm x}}{3}\left(M_{i \pm 1, j, k}-M_{i \pm 2, j, k}\right) \\
& \ell_{i, j, k}^{2^{ \pm}}=M_{i, j \pm 1, k}+\frac{s_{i, j \pm 1, k}^{ \pm y}}{3}\left(M_{i, j \pm 1, k}-M_{i, j \pm 2, k}\right) \\
& \ell_{i, j, k}^{3^{ \pm}}=M_{i, j, k \pm 1}+\frac{s_{i, j, k \pm 1}^{ \pm z}}{3}\left(M_{i, j, k \pm 1}-M_{i, j, k \pm 2}\right)
\end{aligned}
$$

Concerning the source term of equation $(5 \mathrm{~b}), \mathcal{S}(M) \Delta \alpha$, we evaluate $\mathcal{S}$ implicitly and discretize the Laplacian operator at second order. From our experience, it is crucial to use a centered scheme as much as possible. This is done in practice by taking into account all finite value neighbours of a point of interest, including those of the NarrowBand, in the calculation of the test value. This feature also affects the switches evaluation in (7). In one space direction, if a left and right values are available, the second derivative is chosen centered, no matter the causality condition in the NarrowBand (i.e. we also use points which are not in the Known set). For this reason, the algorithm is not a fast-marching method in the strictest sense, and we say it has fast-marching like properties.

More precisely, $\left.(\mathcal{S}(M) \Delta \alpha)\right|_{i, j, k}=\mathcal{S}(m) \sum_{\nu=1}^{3} \hat{\Delta}_{\nu}$, where we express only the first term for the sake of simplicity

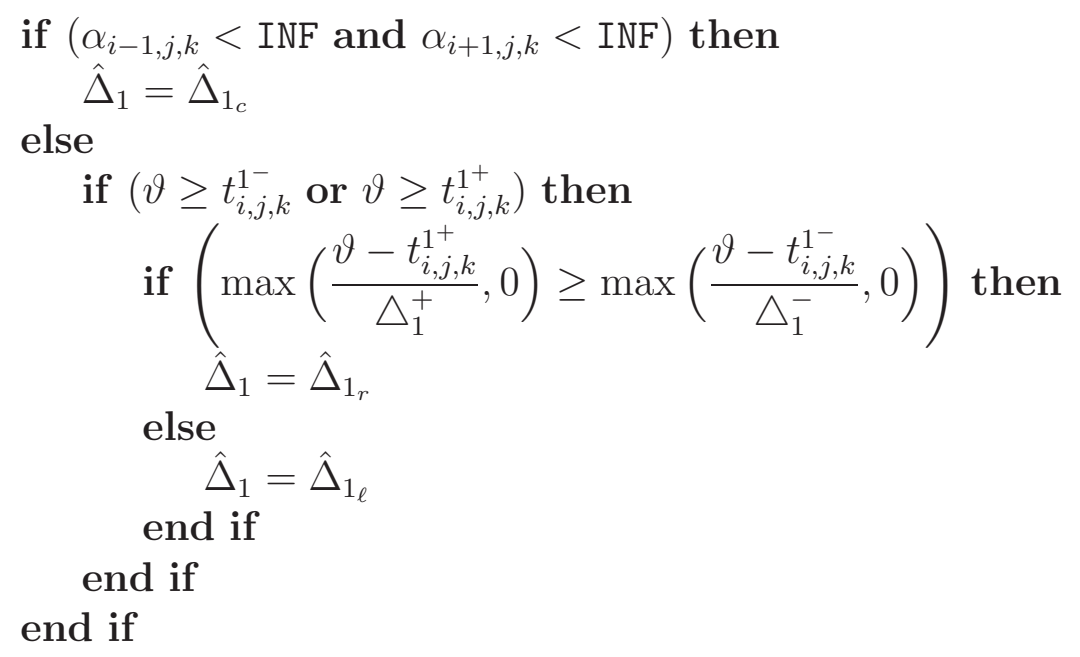


with

- $\hat{\Delta}_{1_{c}}=\frac{\alpha_{i+1, j, k}-2 \vartheta+\alpha_{i-1, j, k}}{\triangle x^{2}}$

- $\hat{\Delta}_{1_{r}}=s_{i, j, k}^{+x} s_{i+1, j, k}^{+x}\left[\frac{\vartheta-2 \alpha_{i+1, j, k}+\alpha_{i+2, j, k}}{\triangle x^{2}}\right.$

$\left.+s_{i+2, j, k}^{+x}\left(\frac{\vartheta-2 \alpha_{i+1, j, k}+\alpha_{i+2, j, k}}{\triangle x^{2}}-\frac{\alpha_{i+3, j, k}-2 \alpha_{i+2, j, k}+\alpha_{i+1, j, k}}{\triangle x^{2}}\right)\right]$

- $\hat{\Delta}_{1_{\ell}}=s_{i, j, k}^{-x} s_{i-1, j, k}^{-x}\left[\frac{\vartheta-2 \alpha_{i-1, j, k}+\alpha_{i-2, j, k}}{\triangle x^{2}}\right.$

$\left.+s_{i-2, j, k}^{-x}\left(\frac{\vartheta-2 \alpha_{i-1, j, k}+\alpha_{i-2, j, k}}{\triangle x^{2}}-\frac{\alpha_{i-3, j, k}-2 \alpha_{i-2, j, k}+\alpha_{i-1, j, k}}{\triangle x^{2}}\right)\right]$

where we recall that INF denotes the huge initial positive value given to points in the Far set.

The final form of the non linear system on $\vartheta$ and $m$ is then

$$
\left\{\begin{array}{l}
\sum_{\nu=1}^{3} \max ^{2}\left(\frac{\vartheta-t_{i, j, k}^{\nu^{-}}}{\triangle_{\nu}^{-}}, \frac{\vartheta-t_{i, j, k}^{\nu^{+}}}{\triangle_{\nu}^{+}}, 0\right)=\frac{1}{m^{2}} \\
\sum_{\nu=1}^{3}\left[\left(\frac{\vartheta-t_{i, j, k}^{\nu^{-}}}{\triangle_{\nu}^{-}}\right)^{+} \frac{m-\ell_{i, j, k}^{\nu^{-}}}{\triangle_{\nu}^{-}}-\left(\frac{t_{i, j, k}^{\nu^{+}}-\vartheta}{\triangle_{\nu}^{+}}\right)^{-} \frac{\ell_{i, j, k}^{\nu^{+}}-m}{\triangle_{\nu}^{+}}\right]=\mathcal{S}(m) \sum_{\nu=1}^{3} \hat{\Delta}_{\nu}
\end{array}\right.
$$

Remark 2.2. As in remark 2.1, the first-order accurate version of the equation (8b) uses the following identities: for the advection part, $\ell_{i, j, k}^{1^{ \pm}}=M_{i \pm 1, j, k}$, $\ell_{i, j, k}^{2^{ \pm}}=M_{i, j \pm 1, k}, \ell_{i, j, k}^{3^{ \pm}}=M_{i, j, k \pm 1}$, and the Laplacien is discretized taking $s_{i \pm 2, j, k}^{ \pm x}=$ $0, s_{i, j \pm 2, k}^{ \pm y}=0$ and $s_{i, j, k \pm 2}^{ \pm z}=0$ in the expressions of $\hat{\Delta}_{1}, \hat{\Delta}_{2}$ and $\hat{\Delta}_{3}$ respectively.

Two strategies have been tested for the numerical resolution of the local nonlinear system (8) on $\vartheta$ and $m$. Given the current approximation $\left(\vartheta^{(p)}, m^{(p)}\right)$, the first approach is a fixed point method which consists in solving the quadratic equation (8a) on $\vartheta$ with $m=m^{(p)}$ to get $\vartheta^{(p+1)}$ which is injected in the equation $(8 \mathrm{~b})$. This gives us a new value $m^{(p+1)}$ by resolution with either a new fixed-point iteration or Newton's method. This procedure 
is repeated until a desired tolerance is met $\left(10^{-6}\right.$ in practice). The second approach is to solve the full coupled system of equations (8) by Newton's procedure. Indeed, the system (8) can be written in the generic form $\mathcal{G}(\mathcal{W})=0$, where $\mathcal{G}$ is a nonlinear function in $\mathcal{W}=(m, \vartheta)^{t}$ which depends on the values of $\alpha$ and $M$ around the point $(i, j, k)$ of the NarrowBand. The resolution of (8) is then done by the following iterative algorithm, for $p \geq 0$,

$$
\mathcal{W}^{(p+1)}=\mathcal{W}^{(p)}-\left(\mathrm{D}_{\mathcal{W}} \mathcal{G}\left(\mathcal{W}^{(p)}\right)\right)^{-1} \mathcal{G}\left(\mathcal{W}^{(p)}\right)
$$

when the computation of the gradient $\mathrm{D}_{\mathcal{W}} \mathcal{G}$ is allowed, and where the starting point $\mathcal{W}^{(0)}$ is determined from the values of $M$ and $\alpha$ on neighboring points. Both approaches have been successfully employed, we did not observe any robustness problem.

\subsection{Boundary conditions}

Two kinds of boundary conditions are commonly required: outgoing and rigid walls. In practice, fictious points are added outside of the computational domain, on which the phase and the Mach number are set. The number of fictious cells depends on the order of the interior numerical scheme.

The outgoing conditions are used on the artificial limits of a free boundary. We impose a huge positive value of $\alpha$ on the fictious points to make the scheme upwind.

When a boundary of the computational domain is bounded by a rigid body $\Gamma_{R}$, a wall condition is used. In this case, a Neumann condition applies: $(\mathbf{n} \cdot \nabla \alpha)_{\Gamma_{R}}=0$. The numerical implementation is done by reflecting the values of $\alpha$ and $M$, by symmetry, on the fictious points. Let us recall here that Whitham's model is able to predict only the irregular shock reflection, namely the Mach reflection or shock-shock singularity in the terminology of Whitham.

\subsection{Summary of the algorithm}

The numerical scheme we designed to solve the GSD model (1) is now summarized. Whitham's model is first rewritten to avoid the direct discretisation of the curvature. The proposed algorithm is an Eulerian approach similar to the fast-marching method performed on a cartesian grid. This algorithm is based on a second order finite difference method and more precisely on an upwind scheme in the direction of the shock propagation with a priority queue acceptance of the local solution. The arrival time and the 
Mach number are obtained by solving a local non-linear system.

The overall structure of our algorithm is close to the FMM one but has two main differences: utilization of points not yet accepted and resolution of the eikonal equation together with the transport equation on the propagation velocity. The fast-marching like algorithm contains two main steps, namely initialisation and iteration, and is organized as follows:

\section{$\Rightarrow$ Initialization step}

Set all points of the computational domain in the Far set by assigning a huge INF value to $\alpha$.

Add each point of the user initial condition in the Known set.

Create the NarrowBand from first neighbours of the Known set.

\section{$\Rightarrow$ Iterative step until the NarrowBand is empty}

Apply boundary conditions.

For each point in the NarrowBand, compute the test values $\vartheta$ and $m$ by solving the local non-linear system (8), by a Newton or fixed point method.

Pick $P_{\min } \equiv\left(i_{\min }, j_{\min }, k_{\min }\right) \in$ NarrowBand such that

$$
\alpha\left(i_{\text {min }}, j_{\text {min }}, k_{\text {min }}\right)=\min _{(i, j, k) \in \text { NarrowBand }} \alpha(i, j, k) .
$$

Add $P_{\min }$ in the Known set/Delete it in NarrowBand.

Add the close neighbors of $P_{\min }$ in NarrowBand if they were in the Far set.

\section{Reference solutions to GSD}

In this section, we provide reference solutions to the GSD equations, that is geometrical configurations where the exact solution can be either calculated analytically or approximated with a quick by-calculation (typically the resolution of a one-dimensional ODE). The numerical solutions of our fast-marching algorithm can then be compared to these reference solutions to evaluate its precision. Ideally, they should cover a wide array of cases, to test the algorithm in the most diverse configurations. Conversely, such reference 
solutions are often hard to provide, and we only expect to determine them in very simple geometrical configurations.

In the case of the GSD equations, it can be noted that infinitely large plane fronts of any normalized velocity $M>1$ are exact solutions of the system in the whole space. Reference solutions can also be found in the radial case, when the configuration is invariant under rotations, and more interestingly, for the Riemann problem in $\mathbb{R}^{2}$. Such solutions enable to test the behaviour of the algorithm on smooth solutions as well as in cases where the velocity has discontinuities. The simplest test case for discontinuous solutions is the compression wedge (see Subsections 3.2.5 and 4.2).

In the remainder of this section we will use the conservative form (1) of the GSD system, with the integral A-M relation

$$
A(M)=A_{0} \exp \left(-\int_{M_{0}}^{M} \frac{m \lambda(m)}{m^{2}-1} d m\right)
$$

where $M_{0}>1$ is the initial condition and $A_{0}$ the corresponding section area. We also introduce the notation

$$
\beta(M)=\frac{M^{2}-1}{\lambda(M)}
$$

The quantity $\beta$ allows to write the results of this section in a simpler manner.

\subsection{Radial solutions}

The simplest configuration in which a solution of the GSD equations can be computed is the radial case where the solution depends only on the radial coordinate $r$. More precisely, in dimension $d=2,3$, the velocity $M$ is a solution of a one-dimensional ODE in the $r$ variable:

$$
\left\{\begin{array}{l}
M(r) \partial_{r} \alpha(r)=1 \\
M(r) \partial_{r} M(r)=-\beta(M(r)) \frac{d-1}{r}
\end{array}\right.
$$

For a given initial condition, this equation can be solved by an high order algorithm to provide a solution of reference, which is helpfull for convergence studies. Analytical solutions are easily obtained in the strong shock limit, $M>>1$. 


\subsection{Solutions of the Riemann problem in dimension 2}

The solutions of the Riemann problem for the GSD equations are of prime importance since they can exhibit discontinuous velocities and develop new intermediate states. The behaviour of the algorithm can then be evaluated on this difficult problem. The simplest test case for discontinuities is the compression wedge, which will be developed in more details later.

For general hyperbolic systems, the theory for the resolution of the Riemann problem only covers the case where the two initial states are not far from each other. Solutions of the Riemann problem for far away initial states are known only for very particular cases, such as the $p$-system. While the GSD equations are in a setting that differs from the usual $p$-system, they can be linked to the $p$-system and indeed the full resolution of the Riemann problem is available.

In his works [28] and [29], Whitham described the form of simple shocks and simple rarefactions. They are studied respectively as the results of the compression of a plane front by a wedge and the diffraction of a plane front by a corner. While these works fully describe the structure of shocks and rarefactions, the rarefaction solution is only given with implicit coordinates. In this part, we describe more explicitly the geometry of the solution. From our knowledge, this is the first time that a comprehensive solution of the GSD Riemann problem is given, together with a link to the $p$-system. Nevertheless, one can mention the works of Henshaw, Smyth and Schwendeman [15] and Schwendeman [20] where the Whitham GSD equations are rewritten in conservative form. Schwendeman [20] also mentions the fact that the Riemann problem admits simple solutions, but does not give full details.

In this section the GSD equations are recasted as a system of $M$ and $\theta$ where $\theta$ parametrizes the front normal as $\mathbf{n}=(\cos \theta) \mathbf{e}_{\mathbf{x}}+(\sin \theta) \mathbf{e}_{\mathbf{y}}$, with $\mathbf{e}_{\mathbf{x}}$ and $\mathbf{e}_{\mathbf{y}}$ the unit vectors along the $\mathrm{x}$ and $\mathrm{y}$ axes respectively. For the Riemann problem, the initial condition is made of two planar fronts as sketched in figure 1: a shock of velocity $M_{\ell}$ and angle $\theta_{\ell}$ interacts with another one of velocity $M_{r}$ and angle $\theta_{r}$. We work in polar coordinates $(\rho, \chi)$, taking $\chi=0$ for the horizontal axis $(O x)$. Note that the initial position of the first front is then the half-line $\left\{\chi=\chi_{\ell}=\theta_{\ell}-\frac{\pi}{2}\right\}$. Similarly, the initial position of the second front is the half-line $\left\{\chi=\chi_{r}=\theta_{r}+\frac{\pi}{2}\right\}$. With these notations, the left front comes before the right one when the angle $\chi$ increases. This is coherent with the standard notations for the theory of the Riemann problem 
and explains why the left front is on the bottom of the picture.

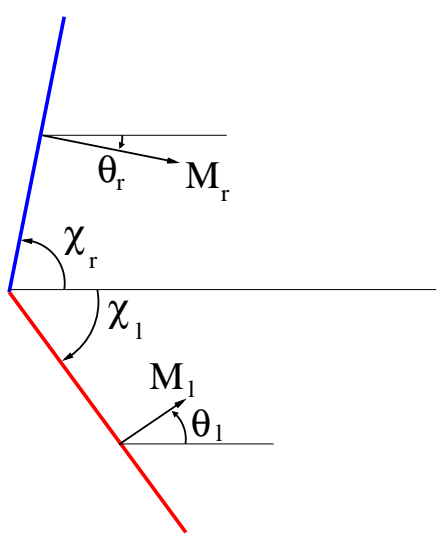

Figure 1: Sketch of the initial condition of the GSD Riemann problem.

\subsubsection{The p-system}

The full resolution of the Riemann problem is known for the $p$-system (see [24], [21]), which can be written in the form

$$
\left\{\begin{array}{ll}
\partial_{t} v-\partial_{x} u & =0 \\
\partial_{t} u+\partial_{x} p(v) & =0
\end{array},\right.
$$

with $t>0, x \in \mathbb{R}$, along with the properties $p^{\prime}<0$ (and $p^{\prime \prime}>0$, but the sign of $p^{\prime \prime}$ plays no role in the resolution of the Riemann problem and is only a result of the usual physical interpretation in terms of isentropic gas dynamics).

As one can note, the $p$-system is one-dimensional in space, and we want to solve the stationary GSD equations in a two-dimensional space. Nevertheless, it can be argued that the level-set function $\alpha$, is a disguised time variable. We then introduce the $(\sigma, \tau)$ system of coordinates where $\mathbf{e}_{\sigma}=\mathbf{n}=\frac{\nabla \alpha}{|\nabla \alpha|}$ is the unit vector normal to the front and $\mathbf{e}_{\tau}$ the unit vector tangent to the front such that $\left(\mathbf{e}_{\sigma}, \mathbf{e}_{\tau}\right)$ is direct. One can note that Whitham [29] already introduced a similar system of coordinates, $\alpha$ and $\beta$, proportional to our $\sigma$ and $\tau$, but with a non normalized gradient, leading to a slightly different system. 
Using the fact that (1) implies

$$
\left\{\begin{array}{l}
\operatorname{curl}\left(\frac{\mathbf{n}}{M}\right)=0 \\
\operatorname{div}\left(\frac{\mathbf{n}}{\mathrm{A}(\mathrm{M})}\right)=0
\end{array}\right.
$$

one can rewrite the GSD system in our system of coordinates as

$$
\begin{cases}\partial_{\sigma} \theta+\partial_{\tau} \log (M) & =0 \\ \partial_{\tau} \theta-\partial_{\sigma} \log (A(M)) & =0\end{cases}
$$

which bears a strong similarity with the $p$-system. Keeping in mind that the setting is different, to write it as a $p$-system we can take

$$
\left\{\begin{array}{l}
u=\theta \\
v=\log (A(M))
\end{array} .\right.
$$

Given that $A$ is a smooth function in $M$ with $A^{\prime}(M)<0$, it is inversible. Noting its inverse by $A^{-1}$, the expression for the function $p$ is

$$
p(v)=\log \left(A^{-1}\left(\mathrm{e}^{v}\right)\right) .
$$

We can then check that

$$
p^{\prime}(v)=\frac{\mathrm{e}^{v}}{A^{\prime}\left(A^{-1}\left(\mathrm{e}^{v}\right)\right) A^{-1}\left(\mathrm{e}^{v}\right)}<0,
$$

because $A^{\prime}<0$, which guarantees that the results obtained in the case of the $p$-system still hold.

\subsubsection{Geometrical structure of simple rarefactions}

A rarefaction is a smooth transition between two constant states $\left(M_{\ell}, \theta_{\ell}\right)$ and $\left(M_{r}, \theta_{r}\right)$ (see for instance figure 2 ). As a consequence of our analysis, we will show that admissible solutions have to satisfy $\partial_{\chi} \theta \geq 0$ and $\theta_{r} \geq \theta_{\ell}$, even if we do not assume it at the beginning of our analysis.

Writing (12) in polar coordinates $(\rho, \chi)$, we get

$$
\left\{\begin{array}{l}
\frac{\partial}{\partial \rho}\left(\frac{\rho \sin (\theta-\chi)}{M}\right)-\frac{\partial}{\partial \chi}\left(\frac{\cos (\theta-\chi)}{M}\right)=0 \\
\frac{\partial}{\partial \rho}\left(\frac{\rho \cos (\theta-\chi)}{A(M)}\right)+\frac{\partial}{\partial \chi}\left(\frac{\sin (\theta-\chi)}{A(M)}\right)=0
\end{array}\right.
$$


Keeping in mind that we are looking for solutions that only depend on the angular variable $\chi$, we see that equations (13) can be rewritten as

$$
\left\{\begin{array}{l}
\left(\partial_{\chi} \theta\right) \sin (\theta-\chi)=-\frac{\left(\partial_{\chi} M\right)}{M} \cos (\theta-\chi) \\
\left(\partial_{\chi} \theta\right) \cos (\theta-\chi)=\left(\partial_{\chi} M\right) \frac{A^{\prime}(M)}{A(M)} \sin (\theta-\chi)=-\left(\partial_{\chi} M\right) \frac{M}{\beta(M)} \sin (\theta-\chi)
\end{array}\right.
$$

Combining the equations of system (14), we get the following fundamental relation

$$
\left(\partial_{\chi} \theta\right)\left(M \sin ^{2}(\theta-\chi)-\frac{\beta(M)}{M} \cos ^{2}(\theta-\chi)\right)=0 .
$$

From this equation we deduce that either $\theta$ is constant (and then $M$ is also constant), which corresponds to the case where the front is planar, or $M$ and $\theta$ satisfy the relation

$$
\tan (\theta-\chi)=\sigma \frac{\sqrt{\beta(M)}}{M} \quad \text { with } \quad \sigma= \pm 1 .
$$

This relation is satisfied for any rarefaction, as soon as $\theta$ is not constant. In particular, we have $\frac{\pi}{2}>\sigma(\theta-\chi)>0$ with $\sigma=1$ for 1-rarefactions, and with $\sigma=-1$ for 2-rarefactions.

The combination of the equations of system (14) also leads to the relation

$$
\left(\partial_{\chi} \theta\right)^{2}=\frac{\left(\partial_{\chi} M\right)^{2}}{\beta(M)}
$$

which gives

$$
\partial_{\chi} \theta=\varepsilon \frac{\left(\partial_{\chi} M\right)}{\sqrt{\beta(M)}} \quad \text { with } \quad \varepsilon= \pm 1
$$

Plugging equations (17) and (15) in system (14) leads to $\sigma \varepsilon=-1$.

We then have

$$
\sigma \omega(M)+\theta=\text { const }
$$

with

$$
\omega(M)=\int_{M_{0}}^{M} \sqrt{\frac{-A^{\prime}(m)}{A(m) m}} d m=\int_{M_{0}}^{M} \frac{1}{\sqrt{\beta(m)}} d m
$$


It can then be shown with other lengthy calculations involving derviations of (15) and (18) with respect to $\chi$, that $M$ satisfies the ODE

$$
\frac{\mathrm{d} M(\chi)}{\mathrm{d} \chi}=-\sigma \mathcal{F}(M(\chi))
$$

along the rarefaction, with $\mathcal{F}(M)=\frac{2 \sqrt{\beta(M)}\left(M^{2}+\beta(M)\right)}{2 M^{2}+M \beta^{\prime}(M)}>0$ (when it is well defined). It can be checked that the denominator of $\mathcal{F}(M)$ is positive when $A^{\prime \prime}(M)>0$ and this last condition can be checked at least numerically on some values of $\gamma$, or in general for large $M$.

In particular for $M(\chi)$ and $\theta(\chi)$ solutions of (20) and (15), the quantity

$$
\sigma \omega(M(\chi))+\theta(\chi)=\text { const }
$$

is independent on $\chi$ (it is indeed a Riemann invariant here). This shows in particular that in any case we have $\partial_{\chi} \theta \geq 0$ and then $\theta_{r} \geq \theta_{l}$. We also deduce from (21) that

$$
\omega\left(M_{\ell}\right)-\omega\left(M_{r}\right)=\sigma\left(\theta_{r}-\theta_{l}\right) .
$$

We can then give the geometrical structure of 1-rarefactions and 2-rarefactions in detail.

\section{Proposition 3.1. (Structure of simple rarefactions)}

Assume that $2 M+\beta^{\prime}(M)>0$. Let $\pi>\theta_{r}-\theta_{l}>0$. We set $\chi_{\ell}=\theta_{\ell}-\frac{\pi}{2}$ and $\chi_{r}=\theta_{r}+\frac{\pi}{2}$.

i) 1-rarefaction

Let $M_{\ell}>M_{r}>1$ satisfying (23) for $\sigma=1$. Then there exist two angles $\chi_{1}^{r-}$ and $\chi_{1}^{r+}$, cf. figure 2(a), satisfying $\chi_{\ell}<\chi_{1}^{r-}<\chi_{1}^{r+}<\chi_{r}-\frac{\pi}{2}$ and such that:

(1) In the sector $\left\{\chi_{\ell} \leq \chi \leq \chi_{1}^{r-}\right\}$, we have $M=M_{\ell}$ and $\theta=\theta_{\ell}$.

(2) In the sector $\left\{\chi_{1}^{r-} \leq \chi \leq \chi_{1}^{r+}\right\}, M$ satisfies (20) and $\theta$ is given by (15) for $\sigma=1$.

(3) In the sector $\left\{\chi_{1}^{r+} \leq \chi \leq \chi_{r}\right\}$, we have $M=M_{r}$ and $\theta=\theta_{r}$.

The angles $\chi_{1}^{r-}$ and $\chi_{1}^{r+}$ are given by (15) for $\sigma=1$, so

$$
\tan \left(\theta_{\ell}-\chi_{1}^{r-}\right)=\frac{\sqrt{\beta\left(M_{\ell}\right)}}{M_{\ell}} \quad \text { and } \quad \tan \left(\theta_{r}-\chi_{1}^{r+}\right)=\frac{\sqrt{\beta\left(M_{r}\right)}}{M_{r}} .
$$


Moreover (21) holds true for $\sigma=1$ for all $\chi \in\left[\chi_{\ell}, \chi_{r}\right]$.

ii) 2-rarefaction

Let $M_{r}>M_{\ell}>1$ satisfying (23) for $\sigma=-1$. Then there exist two angles $\chi_{2}^{r-}$ and $\chi_{2}^{r+}$, cf. figure 2(b), satisfying $\chi_{\ell}+\frac{\pi}{2}<\chi_{2}^{r-}<\chi_{2}^{r+}<\chi_{r}$ and such that:

(1) In the sector $\left\{\chi_{\ell} \leq \chi \leq \chi_{2}^{r-}\right\}$, we have $M=M_{\ell}$ and $\theta=\theta_{\ell}$.

(2) In the sector $\left\{\chi_{2}^{r-} \leq \chi \leq \chi_{2}^{r+}\right\}$, M satisfies (20) and $\theta$ is given by (15) for $\sigma=-1$.

(3) In the sector $\left\{\chi_{2}^{r+} \leq \chi \leq \chi_{r}\right\}$, we have $M=M_{r}$ and $\theta=\theta_{r}$.

The angles $\chi_{2}^{r-}$ and $\chi_{2}^{r+}$ are given by (15) for $\sigma=-1$, so

$$
\tan \left(\theta_{\ell}-\chi_{2}^{r-}\right)=-\frac{\sqrt{\beta\left(M_{\ell}\right)}}{M_{\ell}} \quad \text { and } \quad \tan \left(\theta_{r}-\chi_{2}^{r+}\right)=-\frac{\sqrt{\beta\left(M_{r}\right)}}{M_{r}} .
$$

Moreover (21) holds true for $\sigma=-1$ for all $\chi \in\left[\chi_{\ell}, \chi_{r}\right]$.

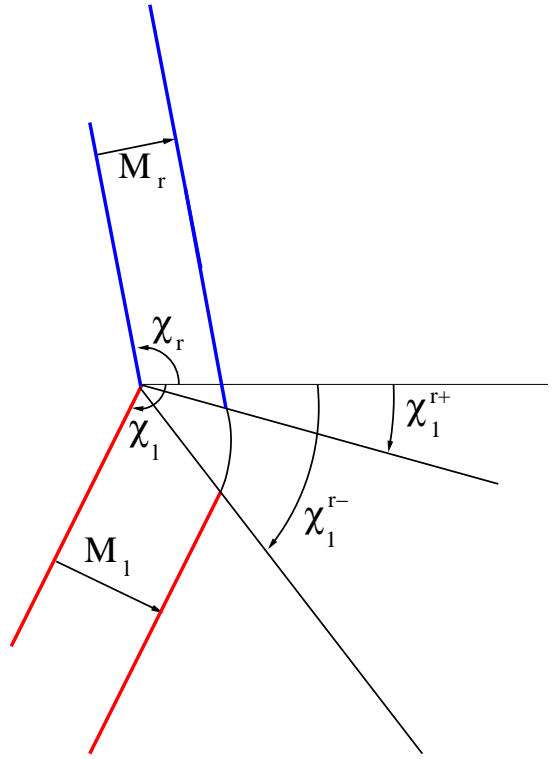

(a) 1-rarefaction

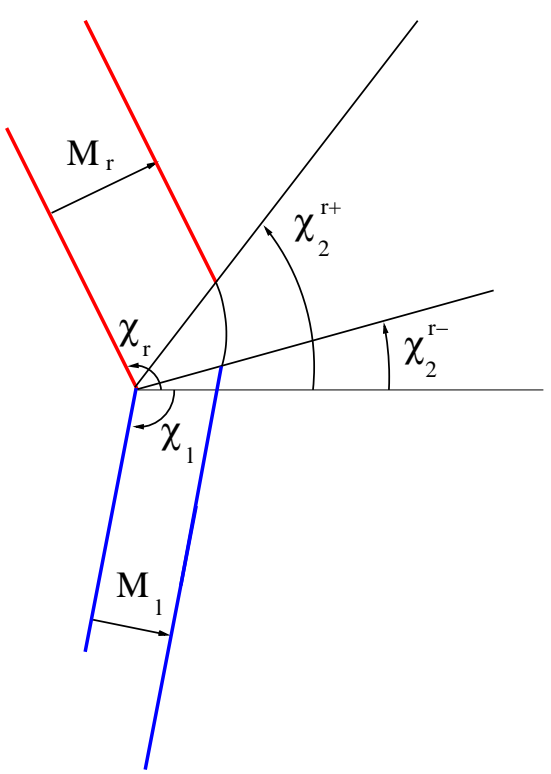

(b) 2-rarefaction

Figure 2: Geometry of simple rarefaction waves. 
Because $M$ satisfies the ODE (20) for $\sigma=1$ along a 1-rarefaction and for $\sigma=-1$ along a 2-rarefaction, independently of $\theta$, the shape of the front along a rarefaction is universal. More precisely if we fix a level-set value $\alpha=t_{0}$, associated with a time $t_{0}>0$, there exists a spiral $\mathcal{S}_{t_{0}}$ of polar equation $\rho=t_{0} \rho_{1}(\chi)$ such that the level set $\left\{\alpha=t_{0}\right\}$ on a rarefaction fan is the image of a portion of $\mathcal{S}_{t_{0}}$ by an isometry (a direct isometry for 1-rarefaction, and an indirect isometry for a 2-rarefaction). Moreover, when the time goes on, the shape of the spiral is simply changed by dilation. Finally this spiral is locally a convex curve, because we have shown that $\partial_{\chi} \theta \geq 0$.

\subsubsection{Geometrical structure of simple shocks}

A shock is a discontinuity between two constant states $\left(M_{\ell}, \theta_{\ell}\right)$ and $\left(M_{r}, \theta_{r}\right)$. Physically, we expect to have $\theta_{r}<\theta_{\ell}$ for a simple shock and indeed, while it is possible to construct functions with a simple shock that are formal solutions of (12) with $\theta_{r}<\theta_{\ell}$, such solutions do not satisfy the entropy condition and are thus not physical (see Remark 3.2). Both quantities $M$ and $\theta$ are discontinuous through the shock, but the level-set $\alpha$ is continuous, albeit not smooth (see figure 3).

The trajectory of the (punctual) shock is the half-line $\chi=\chi^{s}$ with $\chi_{\ell}<$ $\chi^{s}<\chi_{r}$. In order to satisfy the divergence equation in (1), we need to have $\chi^{s}-\chi_{\ell}<\pi$ and $\chi_{r}-\chi^{s}<\pi$ and to have either $\chi_{\ell}<\chi^{s}<\chi_{r}-\frac{\pi}{2}$ or $\chi_{\ell}+\frac{\pi}{2}<\chi^{s}<\chi_{r}$. Otherwise, the flux of $\frac{\mathbf{n}}{A}$ would be entering the shock from both sides, which would contradict the fact that $\frac{\mathbf{n}}{A}$ is divergence free.

When $\chi_{\ell}<\chi^{s}<\chi_{r}-\frac{\pi}{2}$, we have a 1-shock and the continuity of $\alpha$ forces $M_{r}>M_{\ell}$. Using system (12),

we can get the relations between $M_{\ell}, M_{r}, \theta_{\ell}, \theta_{r}$ and $\chi^{s}=\chi_{1}^{s}$ with $\theta_{\ell}, \theta_{r}>$ $\chi_{1}^{s}$. The first equation and the continuity of $\alpha$ lead to

$$
\frac{M_{\ell}}{\cos \left(\theta_{\ell}-\chi^{s}\right)}=\frac{M_{r}}{\cos \left(\theta_{r}-\chi^{s}\right)} .
$$

The second equation gives that the flux of $\frac{\mathbf{n}}{A(M)}$ through the boundary of a closed domain is zero. If we consider the flux for a tube going through the interface between the two states, we get

$$
\frac{A\left(M_{\ell}\right)}{\sin \left(\theta_{\ell}-\chi^{s}\right)}=\frac{A\left(M_{r}\right)}{\sin \left(\theta_{r}-\chi^{s}\right)} \text {. }
$$


From (23) and (24) we deduce

$$
\tan \left(\theta_{r}-\chi^{s}\right)=\frac{A\left(M_{r}\right) \sin \left(\theta_{\ell}-\theta_{r}\right)}{A\left(M_{\ell}\right)-A\left(M_{r}\right) \cos \left(\theta_{\ell}-\theta_{r}\right)}=-\frac{M_{\ell}-M_{r} \cos \left(\theta_{\ell}-\theta_{r}\right)}{M_{r} \sin \left(\theta_{\ell}-\theta_{r}\right)},
$$

and then by symmetry $r / \ell$, we get

$$
\tan \left(\theta_{\ell}-\chi^{s}\right)=-\frac{A\left(M_{\ell}\right) \sin \left(\theta_{\ell}-\theta_{r}\right)}{A\left(M_{r}\right)-A\left(M_{\ell}\right) \cos \left(\theta_{\ell}-\theta_{r}\right)}=\frac{M_{r}-M_{\ell} \cos \left(\theta_{\ell}-\theta_{r}\right)}{M_{\ell} \sin \left(\theta_{\ell}-\theta_{r}\right)} .
$$

Notice that this implies

$$
\cos \left(\theta_{\ell}-\theta_{r}\right)=\frac{A\left(M_{\ell}\right) M_{\ell}+A\left(M_{r}\right) M_{r}}{A\left(M_{r}\right) M_{\ell}+A\left(M_{\ell}\right) M_{r}} .
$$

We notice in particular that the general reordering inequality holds: $a^{+} b^{+}+$ $a^{-} b^{-} \geq a^{+} b^{-}+a^{-} b^{+}$for all $a^{+} \geq a^{-} \geq 0$ and $b^{+} \geq b^{-} \geq 0$. Therefore, the fact that $A(M)$ is decreasing in $M$ implies in particular that

$$
0<\frac{A\left(M_{\ell}\right) M_{\ell}+A\left(M_{r}\right) M_{r}}{A\left(M_{r}\right) M_{\ell}+A\left(M_{\ell}\right) M_{r}} \leq 1
$$

which makes sense for equality (27).

When $\chi_{\ell}+\frac{\pi}{2}<\chi^{s}<\chi_{r}$, we have a 2-shock and the continuity of $\alpha$ forces $M_{r}<M_{\ell}$. Moreover, we have $\theta_{\ell}, \theta_{r}<\chi^{s}=\chi_{2}^{s}$. The same reasoning leads to (23) and (24) which gives the same relations as the ones above.

Remark 3.2. Recall that admissible shocks have to satisfy an entropy condition in order to be stable: this is the Liu E-condition (which implies the Lax E-condition), see paragraph 8.4 in [13]. It is also known that Lax E-condition selects only half of the shock curve (see page 244, paragraph $8.3 \mathrm{in}$ [13]). We can see it on figure 4. This shows that only the case $\theta_{r}<\theta_{\ell}$ is selected for shocks.

With these informations in mind, we can give the structure of simple shocks:

Proposition 3.3. (Structure of simple shocks)

Let $0<\theta_{\ell}-\theta_{r}<\frac{\pi}{2}$ and $M_{r}, M_{\ell}>1$ satysfying (27).

i) 1-shock 
If $M_{r}>M_{\ell}>1$, then there exists $\chi^{s}=\chi_{1}^{s}<\theta_{\ell}, \theta_{r}$ given by (25) (or equivalently by (26)), such that the situation is pictured on figure 3(a).

ii) 2-shock

If $M_{\ell}>M_{r}>1$, then there exists $\chi^{s}=\chi_{2}^{s}>\theta_{\ell}, \theta_{r}$ given by by (25) (or equivalently by (26)), such that the situation is pictured on figure 3(b).

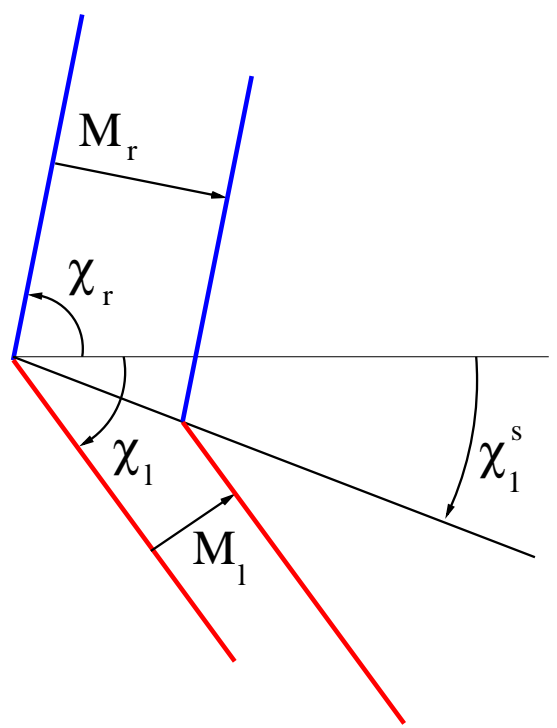

(a) 1-shock

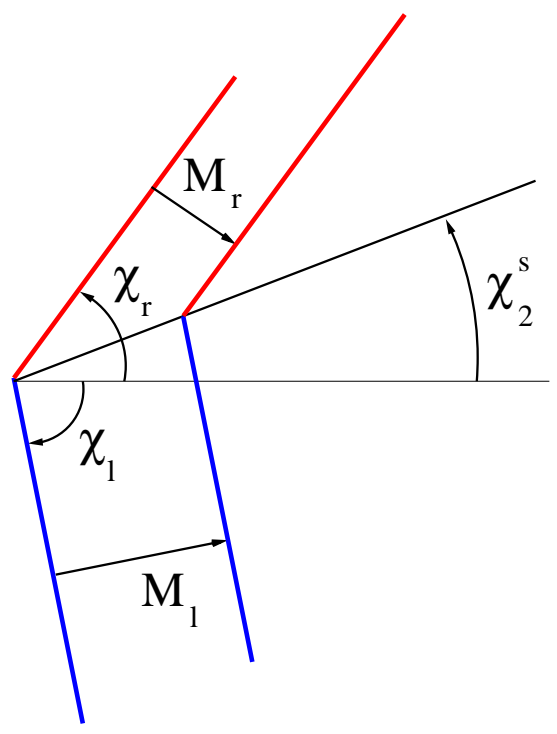

(b) 2-shock

Figure 3: Geometry of simple shock waves.

Remark 3.4. We note that by replacing $\cos \left(\theta_{\ell}-\theta_{r}\right)$ by its expression (27) in (26), one checks that (26) is equivalent to the relation given in [29] for a compression wedge.

$$
\tan \left|\theta_{\ell}-\chi^{s}\right|=\frac{A_{\ell}}{M_{\ell}}\left(\frac{M_{r}^{2}-M_{\ell}^{2}}{A_{\ell}^{2}-A_{r}^{2}}\right)^{1 / 2} \quad \text { with } \quad A_{b}=A\left(M_{b}\right) \quad \text { for } \quad b=\ell, r .
$$

Remark 3.5. Here, we have privileged the geometric interpretation of the Riemann problem. However, one can use the conservative form (13) of the GSD model in polar coordinates to obtain in similar manner as in [29] the equations (26)-(27) and (25)-(27). 


\subsubsection{Complete solutions of the Riemann problem}

The relations satisfied by the velocities $M_{\ell}$ and $M_{r}$ and the angles $\theta_{\ell}$ and $\theta_{r}$ for rarefactions and for shocks lead us to conclude that, as in the case of the $p$-system, there always exists a unique solution of the Riemann problem constituted of at most two transitions (a transition being a rarefaction or a shock). This happens to be true in a whole domain except in a region where the model ceases to be physical (see figure 4).

Supposing the left state $\left(M_{\ell}, \theta_{\ell}\right)$ is fixed, we can define the 1-shock and 2-shock curves functions, $S_{\left(M_{\ell}, \theta_{\ell}\right)}^{1}(M)$ and $S_{\left(M_{\ell}, \theta_{\ell}\right)}^{2}(M)$, by stipulating that for $M>M_{\ell}, \theta=S_{\left(M_{\ell}, \theta_{\ell}\right)}^{1}(M)$ is the only angle such that $\left(M_{\ell}, \theta_{\ell}\right)$ and $(M, \theta)$ satisfy the shock relation (27) (which implies that there is an acceptable 1-shock between the states $\left(M_{\ell}, \theta_{\ell}\right)$ and $\left.(M, \theta)\right)$ and for $M<M_{\ell}, \theta=S_{\left(M_{\ell}, \theta_{\ell}\right)}^{2}(M)$ is the only angle such that $\left(M_{\ell}, \theta_{\ell}\right)$ and $(M, \theta)$ satisfy the shock relation (27) (which implies that there is an acceptable 2-shock between the states $\left(M_{\ell}, \theta_{\ell}\right)$ and $\left.(M, \theta)\right)$. We define $R_{\left(M_{\ell}, \theta_{\ell}\right)}^{1}(M)$ and $R_{\left(M_{\ell}, \theta_{\ell}\right)}^{2}(M)$ in a similar manner. For $M<M_{\ell}, \theta=R_{\left(M_{\ell}, \theta_{\ell}\right)}^{1}(M)$ is the only angle such that $\left(M_{\ell}, \theta_{\ell}\right)$ and $(M, \theta)$ satisfy the 1-rarefaction relation (23) for $\sigma=1$, and for $M>M_{\ell}$, $\theta=R_{\left(M_{\ell}, \theta_{\ell}\right)}^{2}(M)$ is the only angle such that $\left(M_{\ell}, \theta_{\ell}\right)$ and $(M, \theta)$ satisfy the 2 -rarefaction relation (23) for $\sigma=-1$. These functions are drawn in a phase diagram on figure 4.

Nine cases, depending on the position of $\left(M_{r}, \theta_{r}\right)$ relatively to $\left(M_{\ell}, \theta_{\ell}\right)$ in this diagram, are then defined. The first four ones are the general cases, containing two transitions and an intermediate state, as in the study of the p-system (see [24], pp. 306-320). The other five cases are limit cases with at most one transition.

Case 1: $\left(M_{r}, \theta_{r}\right)$ is in zone I

The solution contains a 1-rarefaction between states $\left(M_{\ell}, \theta_{\ell}\right)$ and $\left(M^{*}, \theta^{*}\right)$, and a 2 -shock between states $\left(M^{*}, \theta^{*}\right)$ and $\left(M_{r}, \theta_{r}\right)$.

Case 2: $\left(M_{r}, \theta_{r}\right)$ is in zone II

The solution contains a 1 -shock between states $\left(M_{\ell}, \theta_{\ell}\right)$ and $\left(M^{*}, \theta^{*}\right)$, and a 2-shock between states $\left(M^{*}, \theta^{*}\right)$ and $\left(M_{r}, \theta_{r}\right)$.

Case 3: $\left(M_{r}, \theta_{r}\right)$ is in zone III

The solution contains a 1 -shock between states $\left(M_{\ell}, \theta_{\ell}\right)$ and $\left(M^{*}, \theta^{*}\right)$, and a 
2-rarefaction between states $\left(M^{*}, \theta^{*}\right)$ and $\left(M_{r}, \theta_{r}\right)$.

Relations (23) and (27) ensure that solutions of this form always exist when $\left(M_{r}, \theta_{r}\right)$ is in zone I, II or III.

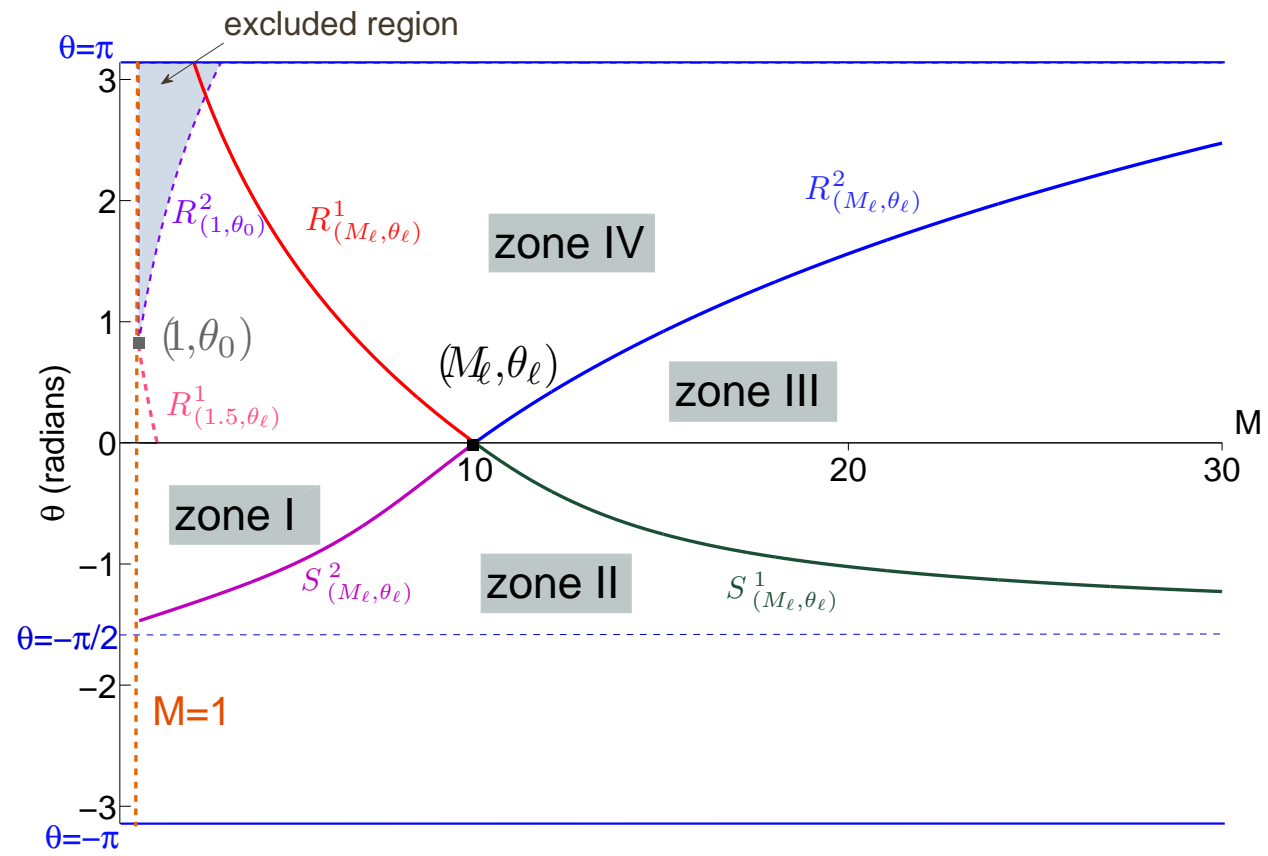

Figure 4: Example of a phase diagram for $\left(M_{\ell}, \theta_{\ell}\right)=(10,0)$ (curves $R_{\left(M_{\ell}, \theta_{\ell}\right)}^{1}, R_{\left(M_{\ell}, \theta_{\ell}\right)}^{2}$, $S_{\left(M_{\ell}, \theta_{\ell}\right)}^{1}$ and $\left.S_{\left(M_{\ell}, \theta_{\ell}\right)}^{2}\right)$, for $\left(M_{\ell}, \theta_{\ell}\right)=(1.5,0)\left(\operatorname{curve} R_{(1.5,0)}^{1}\right)$ and for $\left(M_{\ell}, \theta_{\ell}\right)=\left(1, \theta_{0}\right)$ (curve $R_{\left(1, \theta_{0}\right)}^{2}$ ).

Case 4: $\left(M_{r}, \theta_{r}\right)$ is in zone $\mathbf{I V}$

Except when $\left(M_{r}, \theta_{r}\right)$ is in a particular, excluded region, there exists a solution containing a 1-rarefaction between states $\left(M_{\ell}, \theta_{\ell}\right)$ and $\left(M^{*}, \theta^{*}\right)$, and a 2-rarefaction between states $\left(M^{*}, \theta^{*}\right)$ and $\left(M_{r}, \theta_{r}\right)$. The curve $R_{\left(M_{\ell}, \theta_{\ell}\right)}^{1}$ intersects the vertical axis at the point $\left(1, \theta_{0}\right)$. The boundary of the excluded region is then given by the curve $\theta=R_{\left(1, \theta_{0}\right)}^{2}(M)$.

In zone $\mathrm{I}$, as the shape of the front along a rarefaction is universal, it is 
possible to find the shape of the front (for example the level set $\left\{\alpha=t_{0}\right\}$ ) by noticing that the intermediate plane front must be both tangent to the (sole) image by a direct isometry of $\mathcal{S}_{t_{0}}$ tangent to the front of the state $\left(M_{\ell}, \theta_{\ell}\right)$ and tangent to the sole image by an indirect isometry of $\mathcal{S}_{t_{0}}$ tangent to the front of the state $\left(M_{r}, \theta_{r}\right)$, where we recall that $\mathcal{S}_{t_{0}}$ was defined at the end of subsection 3.2.2. In particular the front is constructed by a simple geometrical procedure (see figure 5).

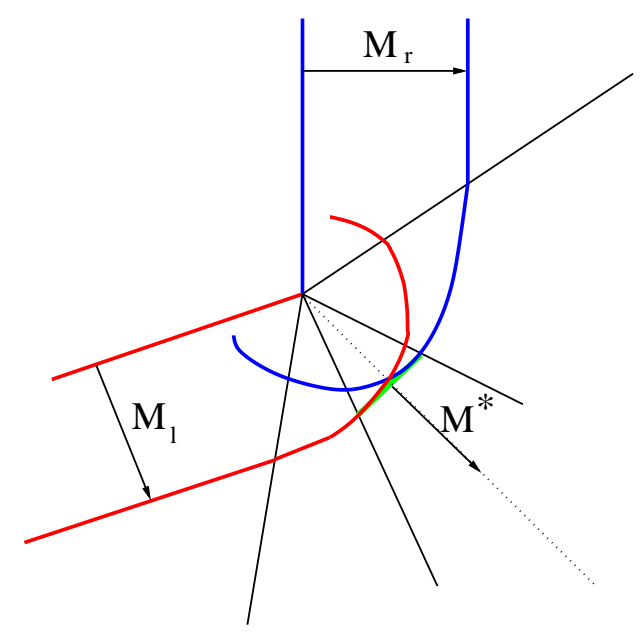

Figure 5: Geometrical determination of the intermediate state for a double rarefaction.

We also remark that as in the analysis of [24], some states in the excluded region (as illustrated in figure 4) can not be joined with $\left(M_{\ell}, \theta_{\ell}\right)$ by the means of two rarefactions. The reason is that $\lim _{M \rightarrow 1} \omega(M)<+\infty$, which means that all curves $R^{1}$ intersect the line $M=1$ at a finite point $\left(1, \theta_{0}\right)$ depending on $\left(M_{\ell}, \theta_{\ell}\right)$. When $M_{\ell}$ is high enough, $\theta_{0} \geq \theta_{\ell}+\pi$ and there is a solution for every physically possible initial data $\left(M_{r}, \theta_{r}\right)$ (that is with $\left.\theta_{r}<\theta_{\ell}+\pi\right)$. But when $M_{\ell}$ is small, $\theta_{0}<\theta_{\ell}+\pi$ and in this case, when $\left(M_{r}, \theta_{r}\right)$ is above the curve $R_{\left(1, \theta_{0}\right)}^{2}$, the Riemann problem does not admit a solution with two rarefactions. The physical interpretation of this is less clear than in the usual interpretation of the $p$-system in terms of isentropic gas dynamics, because the GSD approximation does not work as well when $M$ is near 1 .

Case 5: $M_{r}>M_{\ell}$ and $\theta_{r}=S_{\left(M_{\ell}, \theta_{\ell}\right)}^{1}\left(M_{r}\right)$ 
The solution only contains a 1 -shock between states $\left(M_{\ell}, \theta_{\ell}\right)$ and $\left(M_{r}, \theta_{r}\right)$.

Case 6: $M_{r}<M_{\ell}$ and $\theta_{r}=S_{\left(M_{\ell}, \theta_{\ell}\right)}^{2}\left(M_{r}\right)$

The solution only contains a 2 -shock between states $\left(M_{\ell}, \theta_{\ell}\right)$ and $\left(M_{r}, \theta_{r}\right)$.

Case 7: $M_{r}<M_{\ell}$ and $\left.\theta_{r}=R_{\left(M_{\ell}, \theta_{\ell}\right.}^{1}\right)\left(M_{r}\right)$

The solution only contains a 1-rarefaction between states $\left(M_{\ell}, \theta_{\ell}\right)$ and $\left(M_{r}, \theta_{r}\right)$.

Case 8: $M_{r}>M_{\ell}$ and $\theta_{r}=R_{\left(M_{\ell}, \theta_{\ell}\right)}^{2}\left(M_{r}\right)$

The solution only contains a 2-rarefaction between states $\left(M_{\ell}, \theta_{\ell}\right)$ and $\left(M_{r}, \theta_{r}\right)$.

Case 9: $M_{r}=M_{\ell}$ and $\theta_{r}=\theta_{\ell}$

The solution is a uniform planar front of characteristics $\left(M_{\ell}, \theta_{\ell}\right)$.

\subsubsection{Geometrical interpretation}

In the preceding subsection we have constructed the complete solutions of the Riemann problem in the phase space before describing their geometry. There is a more geometrical way to view this reconstruction. Indeed, as it is described by Whitham in [29], a simple shock is the result of the compression of a plane front by a wedge, and a rarefaction the result of the diffraction of a plane front by a corner. Such solutions involve a wall boundary, and in both cases the solution is a plane front near the wall, with a normal vector $\mathbf{n}$ parallel to the wall. As a consequence, two such solutions can be glued together along the wall boundary to form a complete solution of (1), provided they have the same velocity $M^{*}$ at the wall. The solution thus constructed is naturally the solution of a Riemann problem.

Conversely, the solution of any Riemann problem can be seen as the reunion of two such half-solutions involving a wedge or a corner. For a typical solution constituted of two transitions with an intermediary state, we can split the solution by a virtual boundary at the angle $\chi_{w}$ for which $\theta=\chi$. Then the restrictions of the solution on both sectors $\left\{\chi<\chi_{w}\right\}$ and $\left\{\chi>\chi_{w}\right\}$ are solutions which arise from the interaction of a plane front with a wedge or with a corner. Figures 6 and 7 illustrate this construction. 


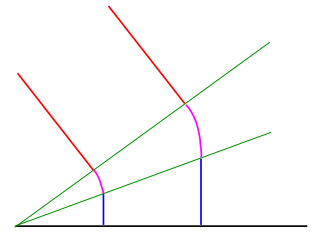

(a) 2-rarefaction

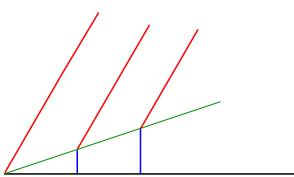

(b) 2-shock

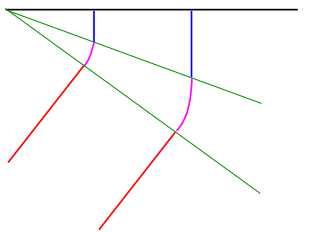

(c) 1-rarefaction

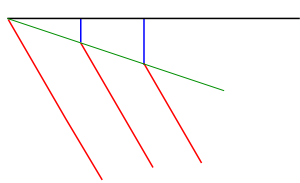

(d) 2-shock

Figure 6: Half-solutions (with a horizontal wall).

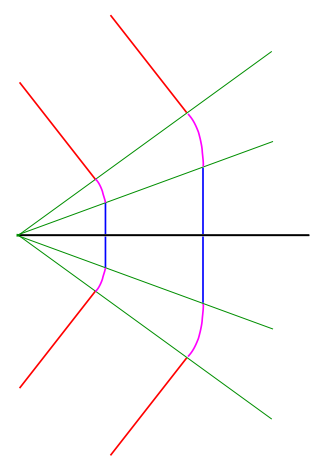

(a)

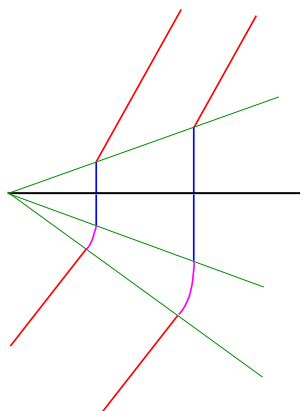

(b)

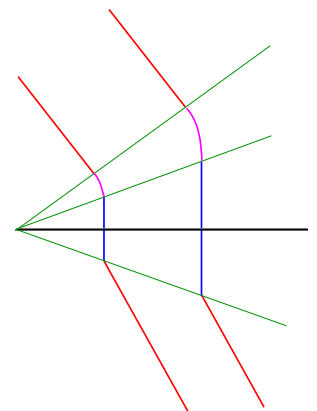

(c)

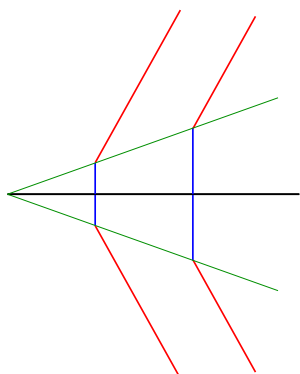

(d)

Figure 7: Complete solutions of the Riemann problem.

\section{Algorithm Validation}

The algorithm summarized in section 2.6 is now evaluated on a set of test cases of increasing complexity. For each of them a reference solution is known or has been introduced previously in section 3. We begin by checking the numerical convergence order of the scheme by comparison with the smooth radial solution, before looking for discontinuous solutions in the case of a compression wedge (shock-shock). The analysis of the Riemann problem completes this experimental study of the numerical scheme. For these reference solutions in the discontinuous case, explicit expressions are developed 
in the strong shock limit (i.e. $M>>1$ ) using the simplified A-M relation

$$
\frac{A}{A_{0}}=\left(\frac{M_{0}}{M}\right)^{\lambda_{\infty}}, \quad \text { with } \lambda_{\infty}=5.0743 \text { for } \gamma=1.4
$$

All numerical experiments are performed with the full A-M relation (9) but for a large enough Mach number value such that the infinite limit is asymptotically reached.

\subsection{Order of the scheme}

The reference solution is obtained by numerically integrating the radial system (10) with a fourth-order Runge-Kutta scheme in cyclindrical and spherical coordinates. The convergence study is made on the discrete $L^{\infty}$ norm.

- Cylindrical shock. For this two dimensional case, the computational domain is reduced to the square $[0,50] \times[0,50]$ due to the symmetry of the problem. The initial conditions on $\alpha$ and $M$ are given by the radial solution (10) on the quarter-circle of radius 4.9 centered at (0.0). We impose $M=10$ for $r<1$ to avoid the singularity at the origin. The boundary conditions are of outgoing type on the edges $\{x=50\}$ and $\{y=50\}$, and of rigid wall type on the edges $\{x=0\}$ and $\{y=0\}$. For this test case, the determination of test values $\vartheta$ and $m$ is made by the fixed-point algorithm.

The tables (1(a)) and (1(b)) give the norm of the error versus the grid spacing for both the first-order and second-order schemes respectively. Note that $N$ is the number of nodes in each direction and that $\triangle x=\triangle y$. The discret order is defined by:

$$
\text { order }=\frac{\log \left(E_{2} / E_{1}\right)}{\log \left(N_{1} / N_{2}\right)}
$$

where $E_{1}$ and $E_{2}$ are respectively the errors associated to the meshes with $N_{1} \times N_{1}$ and $N_{2} \times N_{2}$ elements. A logarithmic representation is given in figures 8(a)-8(b). One can note that the expected order of convergence is recovered and as expected, the second-order scheme provides a better accuracy level. 
(a) First-order

\begin{tabular}{|c||c||c||c||c|}
\hline Mesh & Error $M$ & Order $M$ & Error $\alpha$ & Order $\alpha$ \\
\hline $100 \times 100$ & $9.2577 \mathrm{e}-02$ & & $1.5226 \mathrm{e}-01$ & \\
$200 \times 200$ & $4.2359 \mathrm{e}-02$ & 1.1280 & $6.7709 \mathrm{e}-02$ & 1.1691 \\
$300 \times 300$ & $2.7900 \mathrm{e}-02$ & 1.0298 & $4.4638 \mathrm{e}-02$ & 1.0276 \\
$400 \times 400$ & $2.0718 \mathrm{e}-02$ & 1.0346 & $3.3047 \mathrm{e}-02$ & 1.0451 \\
$500 \times 500$ & $1.6447 \mathrm{e}-02$ & 1.0346 & $2.6159 \mathrm{e}-02$ & 1.0475 \\
$600 \times 600$ & $1.3723 \mathrm{e}-02$ & 0.9930 & $2.1880 \mathrm{e}-02$ & 0.9797 \\
$800 \times 800$ & $1.0302 \mathrm{e}-02$ & 0.9968 & $1.6198 \mathrm{e}-02$ & 1.0452 \\
$900 \times 900$ & $9.1333 \mathrm{e}-03$ & 1.0222 & $1.4450 \mathrm{e}-02$ & 0.9692 \\
$1000 \times 1000$ & $8.1830 \mathrm{e}-03$ & 1.0427 & $1.2935 \mathrm{e}-02$ & 1.0514 \\
\hline E.C.O. ${ }^{1}$ & \multicolumn{2}{||}{1.0445} & \multicolumn{2}{|c|}{1.0597} \\
\hline
\end{tabular}

(b) Second-order

\begin{tabular}{|c||c||c||c||c|}
\hline Mesh & Error $M$ & Order $M$ & Error $\alpha$ & Order $\alpha$ \\
\hline $100 \times 100$ & $1.2571 \mathrm{e}-02$ & & $1.6974 \mathrm{e}-02$ & \\
$200 \times 200$ & $2.8049 \mathrm{e}-03$ & 2.1641 & $3.7407 \mathrm{e}-03$ & 2.1819 \\
$300 \times 300$ & $1.2828 \mathrm{e}-03$ & 1.9295 & $1.6097 \mathrm{e}-03$ & 2.0797 \\
$400 \times 400$ & $7.1314 \mathrm{e}-04$ & 2.0409 & $8.8050 \mathrm{e}-04$ & 2.0971 \\
$500 \times 500$ & $4.6273 \mathrm{e}-04$ & 1.9383 & $5.5824 \mathrm{e}-04$ & 2.0422 \\
$600 \times 600$ & $3.0824 \mathrm{e}-04$ & 2.2284 & $3.8439 \mathrm{e}-04$ & 2.0466 \\
$800 \times 800$ & $1.5767 \mathrm{e}-04$ & 2.3303 & $2.0961 \mathrm{e}-04$ & 2.1080 \\
$900 \times 900$ & $1.2552 \mathrm{e}-04$ & 1.9358 & $1.6077 \mathrm{e}-04$ & 2.2522 \\
$1000 \times 1000$ & $1.0399 \mathrm{e}-04$ & 1.7857 & $1.3119 \mathrm{e}-04$ & 1.9299 \\
\hline E.C.O. & \multicolumn{2}{|c|}{2.0819} & \multicolumn{2}{|c|}{2.1047} \\
\hline
\end{tabular}

Table 1: Mesh convergence in the $L^{\infty}$ norm for the cylindrical case.

\footnotetext{
${ }^{1}$ E.C.O. denotes the Experimental Convergence Order given by the linear fit of the error in the logarithmic scale.
} 


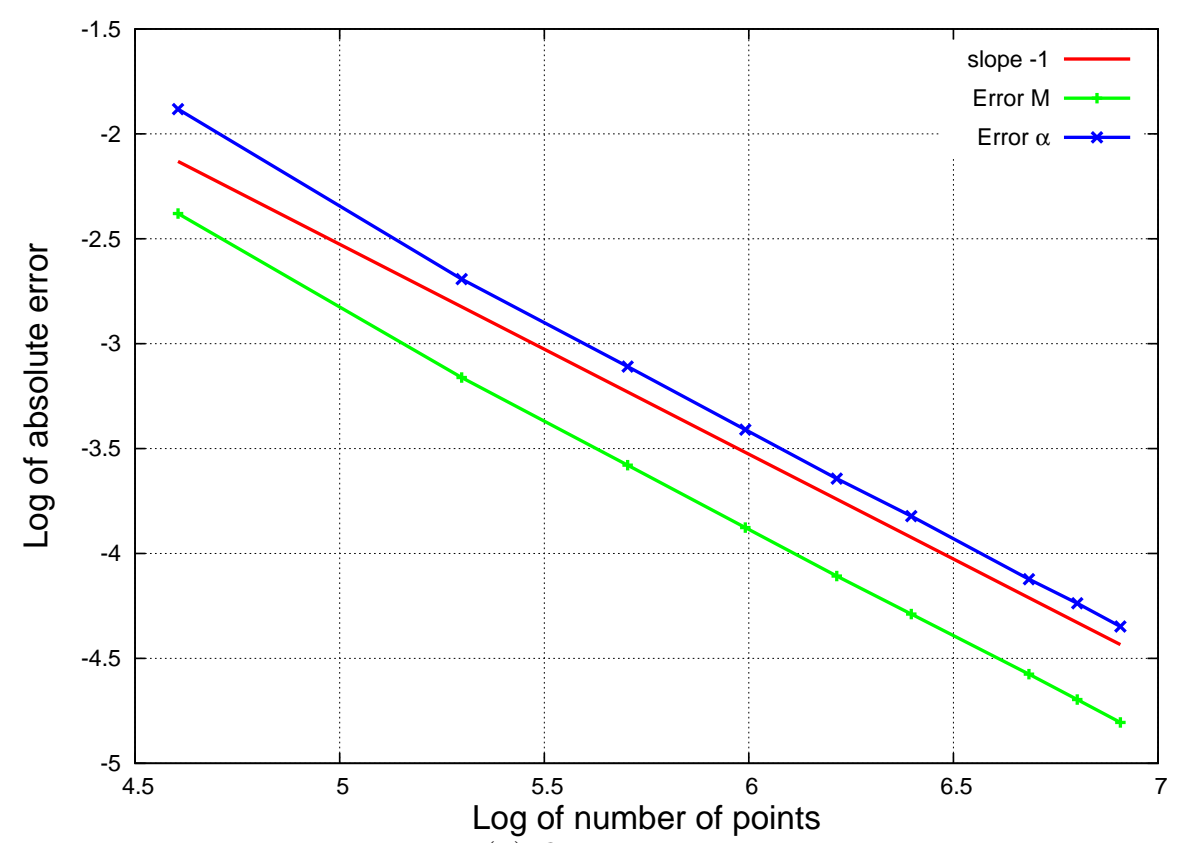

(a) first-order

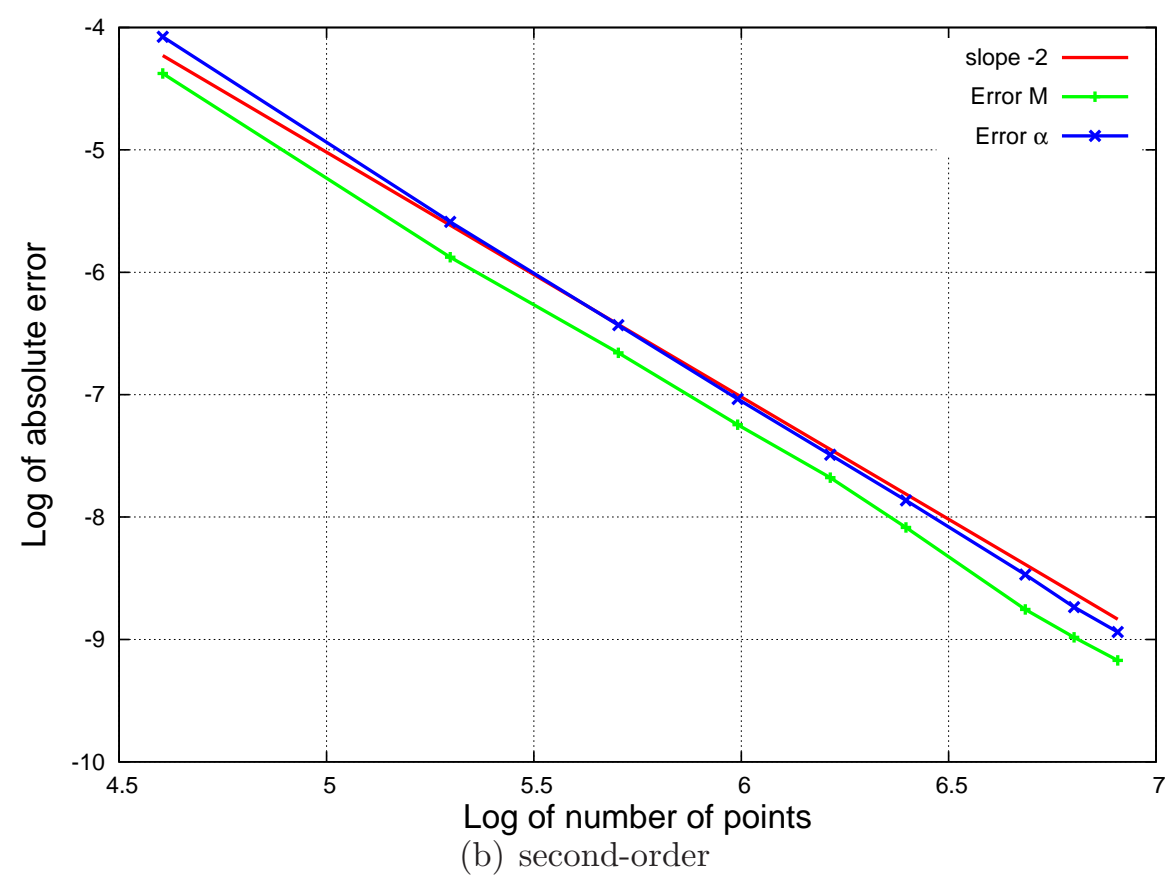

Figure 8: Mesh convergence in the $L^{\infty}$ norm for the cylindrical case in the logarithmic scale. 
- Spherical shock. The analysis of the numerical order of the scheme is also carried on in 3D. Using symmetry conditions, the computational domain reduces to the cube $[0,10] \times[0,10] \times[0,10]$. The initial front is given by the radial solution on the sphere of radius 2 centered at the origin. As in the $2 \mathrm{D}$ case, we impose $M=10$ for $r<1$ to avoid the singularity at the origin. The boundary conditions are of outgoing type on the edges $\{x=10\}$, $\{y=10\},\{z=10\}$, and of rigid wall type on the edges $\{x=0\},\{y=0\}$, $\{z=0\}$. Here, the resolution of the nonlinear system (8) is made by Newton's method. The results of the second-order numerical scheme are compared with the semi-analytical radial solution considered previously. The table 2 gives the error in the discrete $L^{\infty}$ norm between the two solutions, the logarithms of these values are displayed in figure 9 . The second-order of convergence is also obtained in 3D.

\begin{tabular}{|c||c||c||c||c|}
\hline Mesh & Error $M$ & Order $M$ & Error $\alpha$ & Order $\alpha$ \\
\hline $40 \times 40 \times 40$ & 0.00557 & & 0.00521 & \\
$60 \times 60 \times 60$ & 0.00234 & 2.1389 & 0.00232 & 1.9953 \\
$80 \times 80 \times 80$ & 0.00129 & 2.0700 & 0.00126 & 2.1220 \\
$100 \times 100 \times 100$ & 0.000791 & 2.1919 & 0.000808 & 1.9911 \\
$160 \times 160 \times 160$ & 0.000309 & 1.9999 & 0.000308 & 2.0520 \\
\hline E.C.O. & \multicolumn{2}{|c||}{2.0895} & \multicolumn{2}{|c|}{2.2873} \\
\hline
\end{tabular}

Table 2: Mesh convergence in the $L^{\infty}$ norm for the spherical case.

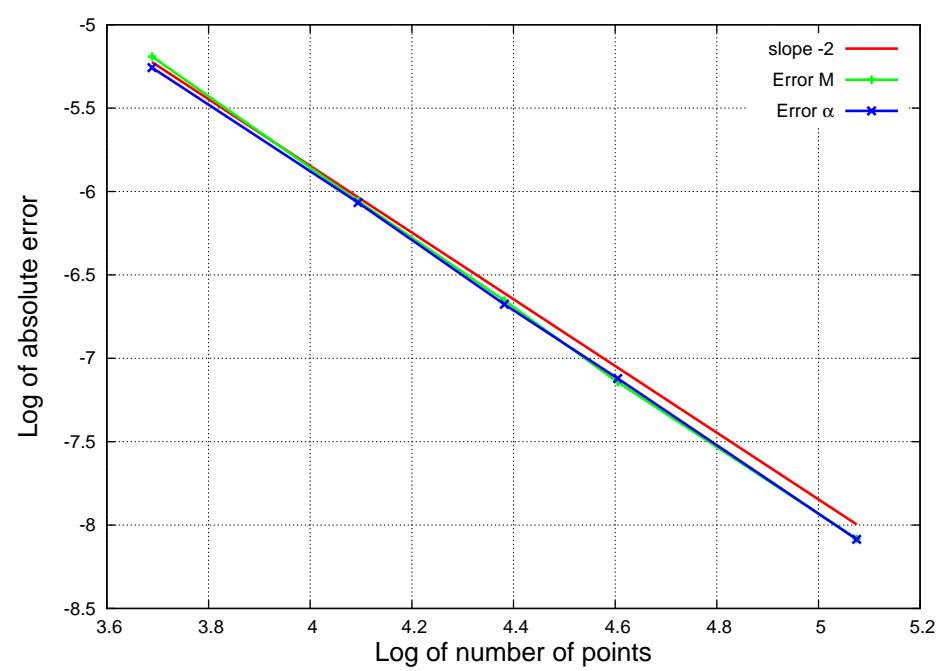

Figure 9: Mesh convergence in the $L^{\infty}$ norm in the logarithmic scale. 
These smooth test cases have proven that our fast-marching like algorithm doesn't suffer from using points in the NarrowBand and that the expected theoretical order of convergence is reached.

\subsection{Compression wedge test case}

Attention is now paid to discontinuous solutions. We deal here with a well-know test case, studied by many authors [29, 2], consisting in the diffraction of an oblique shock by a wedge. It can be seen as an application of the study of simple shocks. For an incoming front of velocity $M_{0}$ and angle $\theta_{0}=\beta$ interacting with a wedge of angle $\theta_{w}=0$, we are looking for a 2 -shock joining the states $\left(M_{\ell}, \theta_{\ell}\right)=\left(M_{w}, 0\right)$ and $\left(M_{r}, \theta_{r}\right)=\left(M_{0}, \beta\right)$, the velocity $M_{w}$ of the Mach front and the angle $\chi_{2}^{s}$ of the trajectory of the triple point being unknown. The analysis of the structure of 2-shocks then gives us that $M_{w}$ is governed by the relation (27), and $\chi_{2}^{s}$ by (25).

In the strong shock limit, the equations (28) and (27) can be simplified in the following implicit relationship between $\beta$ and $\chi_{2}^{s}$

$$
\left\{\begin{aligned}
\tan \chi_{2}^{s} & =m^{\lambda_{\infty}}\left(\frac{1-m^{2}}{1-m^{2 \lambda_{\infty}}}\right)^{1 / 2} \\
\cos \beta & =\frac{m+m^{\lambda_{\infty}}}{1+m^{1+\lambda_{\infty}}}
\end{aligned}\right.
$$

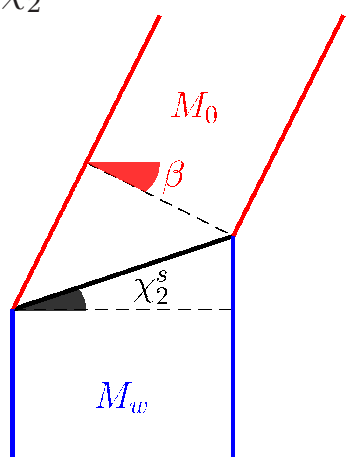

where $m=\frac{M_{0}}{M_{w}}<1$ denotes the Mach number ratio on either side of the shock-shock and $\lambda_{\infty}=5.0743$. The subscripts $w$ and 0 characterize the quantities on the wall and those of the incident (or initial) shock respectively, $\chi_{2}^{s}$ is the angle of the shock-shock line with the horizontal axis $(O x)$. This analytical solution is displayed as a solid curve in the figure 10 .

Here, the wedge is chosen aligned with the $(O x)$ axis to avoid a difficult boundary treatment. The rigid wall condition is then imposed on the edge $\{y=0\}$. The computation was performed on the domain $[0,3] \times[0,3]$ with a $300 \times 300$ mesh. The initial shock position is given by

$$
\alpha_{0}(x, y)=\frac{(x-0.5) \cos \beta-y \sin \beta}{M_{0}},
$$


where $\beta \in[0, \pi / 2]$ is the angle between the front normal and the wedge, and $M_{0}=10$. The values of $M$ and $\alpha$ for all points inside the half space on the left of the initial shock position $\left(\right.$ i.e. $\left.\alpha_{0}(x, y) \leq 0\right)$ are already intercepted by the front and easily known since the shock is plane. An outflow condition is imposed on the $\{x=3\}$ edge. Special care must be taken for the $\{y=3\}$ edge since it corresponds to an inflow condition, so we set the exact solution on it.

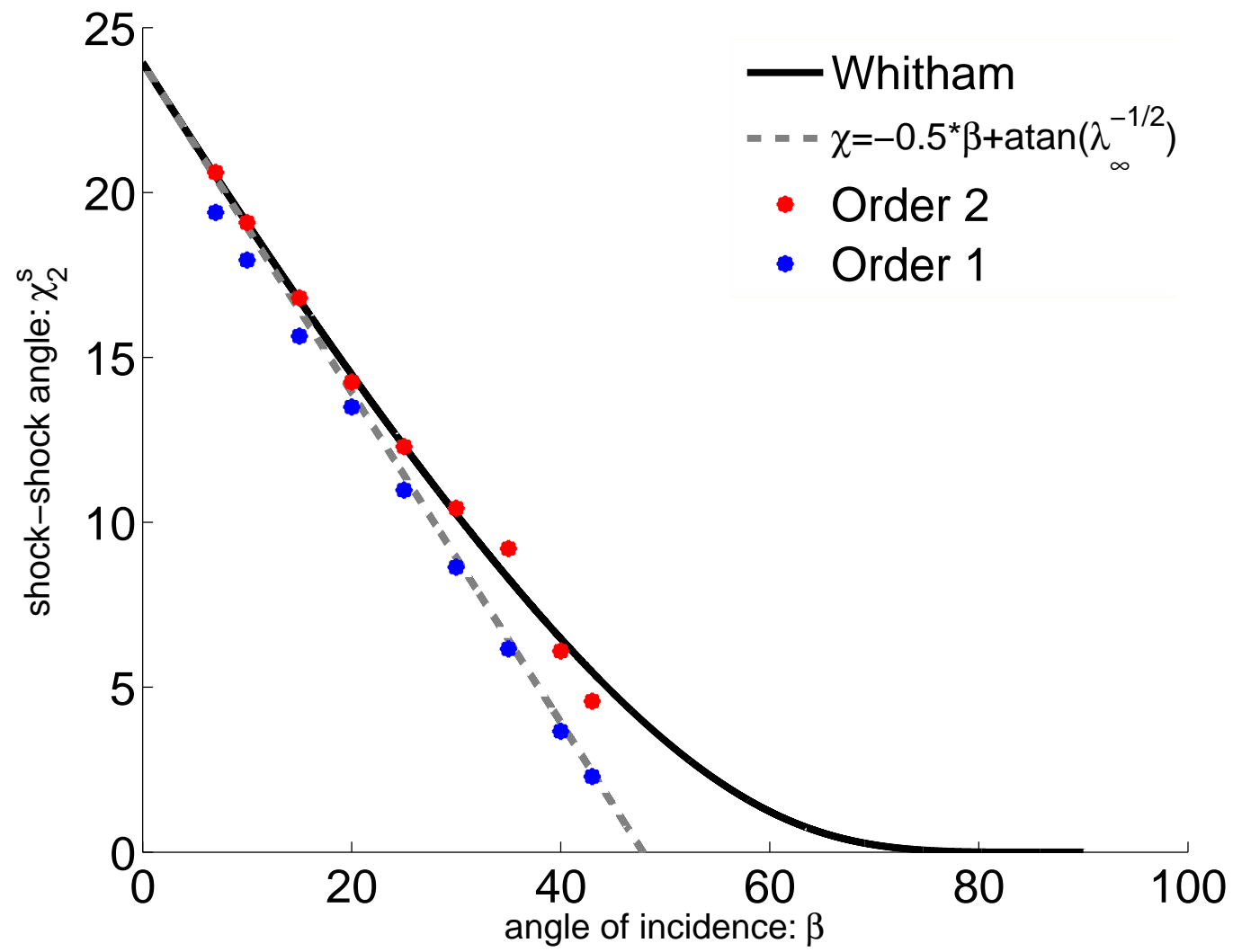

Figure 10: Shock-shock angle versus angle of incidence for the first and second order algorithm in comparison to Whitham's analytical solution.

For a range of incident shock angle, a comparison between this theoretical shock-shock locus and the numerical ones obtained by the first and second order schemes is shown in figure 10. It is worth noting the very good agree- 
ment between the theoretical position and the results of the second-order version. This result is in agreement with [2] where a classical level-set algorithm was used. One can also notice that the first-order numerical scheme underestimates the value of $\chi_{2}^{s}$ by several degrees, and follows a nearly linear behaviour, lying on the tangent (dashed line) to the theoretical curve at $M=1$. A mesh refinement does not improve these results, which demonstrates the necessity to use the second-order scheme when a shock singularity is expected. Above nearly 45 degrees, the second order algorithm is also in default and doesn't show any shock-shock angle. This may be due to the switching procedure, applied as it on the wall, which reduces locally the order of the scheme. A better boundary treatment could help improve this behaviour. Nevertheless, this is not a severe limitation in pratice since the GSD model is not expected to give accurate results in such an extreme case [29].

\subsection{Test cases for the Riemann problem}

A series of numerical experiments is now conducted to assess in more details the ability of the numerical scheme to solve the two dimensional GSD Riemann problem. In section 3 we have given the mathematical solutions and we refer the reader to Appendix A for the detail of their explicit construction in the strong shock limit. We compare these reference solutions to the numerical ones, by taking varying values of the initial constant states to cover all configurations of paragraph 3.2.4. The first tests use the left state $\left(M_{\ell}, \theta_{\ell}\right)$ connected directly to the right state $\left(M_{r}, \theta_{r}\right)$ by an elementary wave (either a shock or a rarefaction), i.e. $\left(M_{r}, \theta_{r}\right)$ lies on one of the curves $\mathrm{R}^{1}$, $\mathrm{R}^{2}, \mathrm{~S}^{1}, \mathrm{~S}^{2}$ issued from $\left(M_{\ell}, \theta_{\ell}\right)$ (see figure 4 ). More general initial datas are then considered to cover the different zones of the figure 4: zone I, zone II, zone $\mathrm{II}$ and zone $\mathrm{I}$, i.e. solutions with an intermediate state.

The numerical solution is obtained on the domain $[0,5] \times[0,5]$ with a $500 \times 500$ grid. In all cases, the left state $\left(M_{\ell}, \theta_{\ell}\right)=(10,0)$ is given and we make the right state $\left(M_{r}, \theta_{r}\right)$ vary in the plane $(M, \theta)$. We display a comparison between the isolines of $\alpha$, obtained by the first and second order schemes, and the exact solution in each configuration. In these figures, the red curve represents the numerical solution, the blue one represents the exact solution and the green lines correspond to the characteristic curves. The following figures gather all the results obtained for the different solutions of the Riemann problems. 


\subsubsection{Simple waves}

Simple waves correspond to cases 5 (1-shock), 6 (2-shock), 7 (1-rarefaction) and 8 (2-rarefraction) of paragraph 3.2.4. They are difficult to compute in practice since numerical roundoff on the initial condition may lead to the emergence of an intermediate state.

The initial right state for the 1-shock and the 2-shock cases are $\left(M_{r}, \theta_{r}\right)=$ $(12,-22.45)$ and $\left(M_{r}, \theta_{r}\right)=(8.5,-20.24)$ respectively. Results are drawn in figures 11 and 12. As expected from the compression wedge study, one can note the excellent behaviour of the second order scheme for the 2-shock case, but also for the 1-shock case. A severe deviation is present for the first order algorithm applied on the 1-shock case.

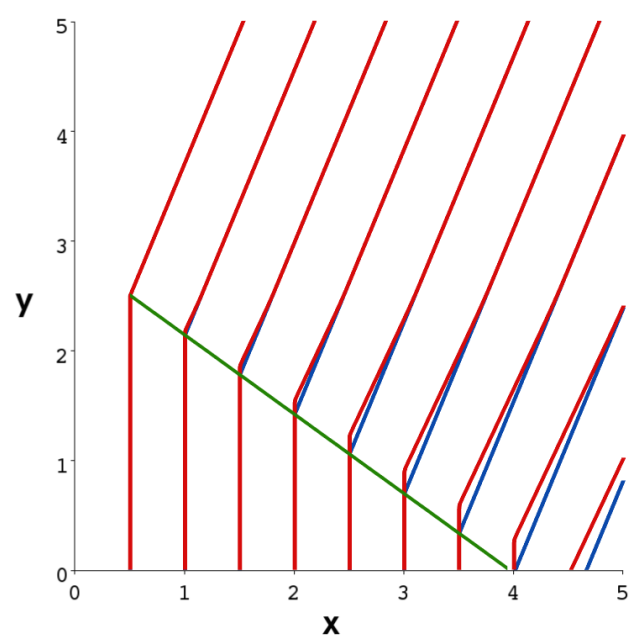

(a) 1 st order

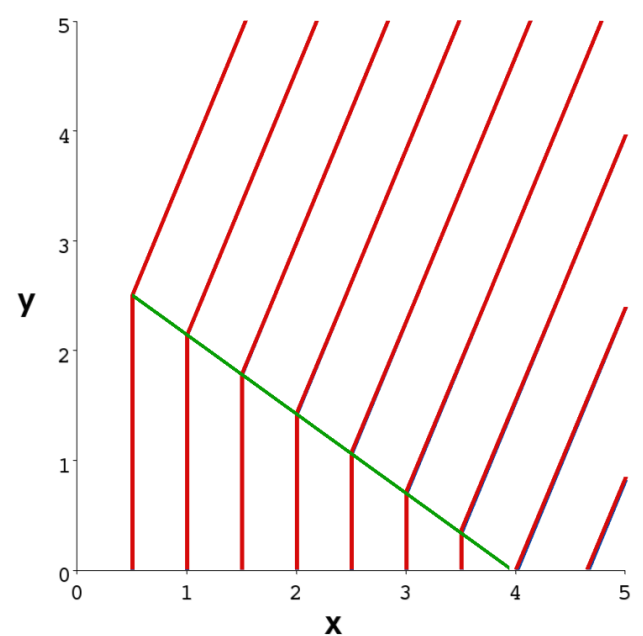

(b) 2nd order

Figure 11: 1-shock case: $\left(M_{\ell}, \theta_{\ell}\right)=(10,0)$ and $\left(M_{r}, \theta_{r}\right)=(12,-22.45)$. Comparison of the numerical solution — and the exact solution — . The characteristic curves — are displayed for convenience. 


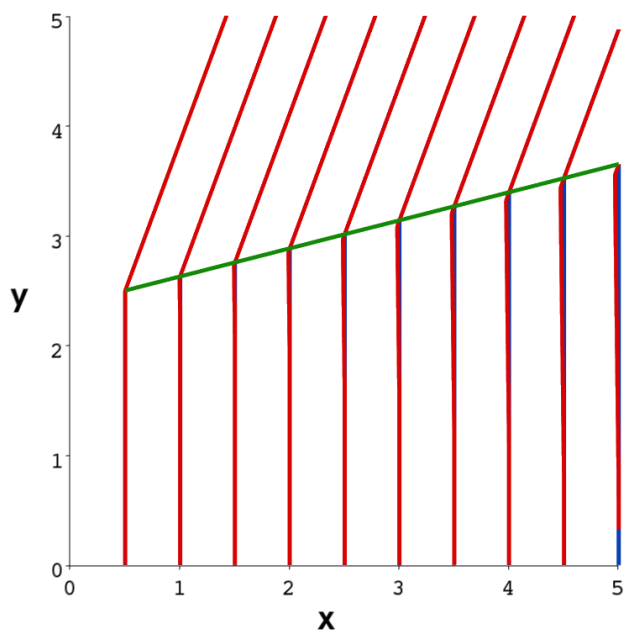

(a) 1 st order

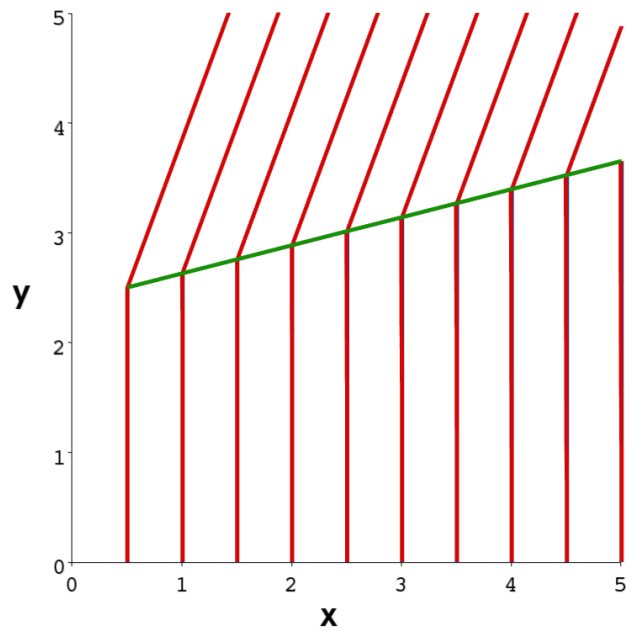

(b) 2nd order

Figure 12: 2-shock case: $\left(M_{\ell}, \theta_{\ell}\right)=(10,0)$ and $\left(M_{r}, \theta_{r}\right)=(8.5,-20.24)$. Same drawing convention.

The initial right state for the 1-rarefaction and the 2-rarefaction cases are $\left(M_{r}, \theta_{r}\right)=(8.5,20.97)$ and $\left(M_{r}, \theta_{r}\right)=(12,23.53)$ respectively. Figures 13 and 14 exhibit a different behaviour for the first order version: the 1-rarefaction wave is well captured while the 2-rarefaction one shows large deviation. The second order scheme is excellent in both cases, nearly superimposed with the reference solution. 


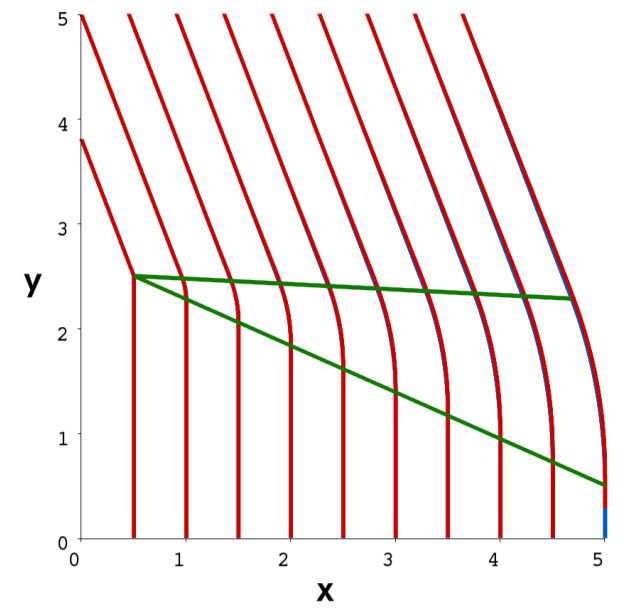

(a) 1st order

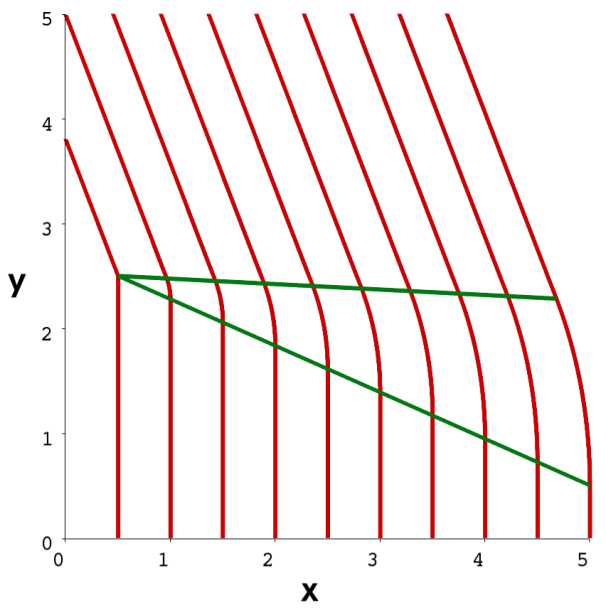

(b) 2nd order

Figure 13: 1-rarefaction case: $\left(M_{\ell}, \theta_{\ell}\right)=(10,0)$ and $\left(M_{r}, \theta_{r}\right)=(8.5,20.97)$. Same drawing convention.

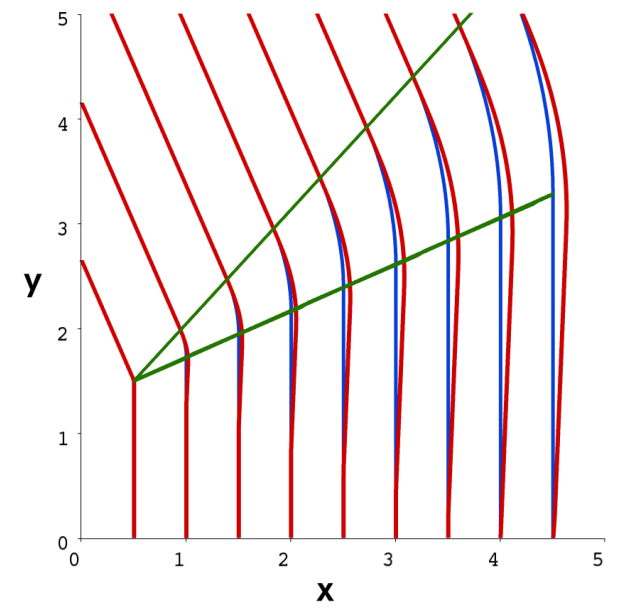

(a) 1st order

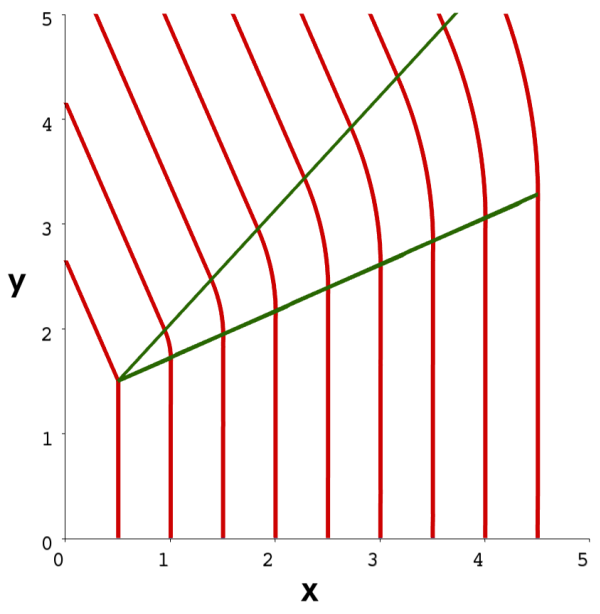

(b) 2nd order

Figure 14: 2-rarefaction case: $\left(M_{\ell}, \theta_{\ell}\right)=(10,0)$ and $\left(M_{r}, \theta_{r}\right)=(12,23.53)$. Same drawing convention.

\subsubsection{1-rarefaction, 2-shock}

This problem corresponds to case 1 of paragraph 3.2.4. The solution consists of a 1-rarefaction connecting the left state $\left(M_{\ell}, \theta_{\ell}\right)=(10,0)$ to a constant intermediate state $\left(M^{*}, \theta^{*}\right)$ followed by a 2 -shock connecting $\left(M^{*}, \theta^{*}\right)$ 
to the right state $\left(M_{r}, \theta_{r}\right)=(5,30)$, i.e. $\left(M_{r}, \theta_{r}\right)$ is within zone I on figure 4 . Numerical results are shown on figure 15 . One can note that the higher order scheme allows a much better capture of the intermediate state, retriving a nearly plane front, while the first order scheme exhibit an unexpected wave pattern.

\subsubsection{1-shock, 2-shock configuration}

This problem corresponds to case 2 of paragraph 3.2.4. The solution consists of a 1-shock connecting the left state $\left(M_{\ell}, \theta_{\ell}\right)=(10,0)$ to a constant intermediate state $\left(M^{*}, \theta^{*}\right)$ followed by a 2 -shock connecting $\left(M^{*}, \theta^{*}\right)$ to the right state $\left(M_{r}, \theta_{r}\right)=(10,-50)$, i.e. $\left(M_{r}, \theta_{r}\right)$ is within zone $\mathbb{I}$ on figure 4 . Numerical results are shown on figure 16. As previously noticed, the higher order scheme allows a better capture of the intermediate state. Here, the first order scheme leads to an underestimation of the intermediate state's Mach number.

\subsubsection{1-shock, 2-rarefaction configuration}

This problem corresponds to case 3 of paragraph 3.2.4. The solution consists of a 1 -shock connecting the left state $\left(M_{\ell}, \theta_{\ell}\right)=(10,0)$ to a constant intermediate state $\left(M^{*}, \theta^{*}\right)$ followed by a 2-rarefaction connecting $\left(M^{*}, \theta^{*}\right)$ to the right state $\left(M_{r}, \theta_{r}\right)=(20,10)$, i.e. $\left(M_{r}, \theta_{r}\right)$ is within zone $\mathbb{I I I}$ on figure 4. Numerical results are shown on figure 17. As expected from the simple wave results, the 1-shock is badly captured by the first order scheme and the overall solution behaviour is dramatically affected. Here again, the second order scheme performs well.

\subsubsection{1-rarefaction, 2-rarefaction configuration}

This problem corresponds to case 4 of paragraph 3.2.4. The solution consists of a 1-rarefaction connecting the left state $\left(M_{\ell}, \theta_{\ell}\right)=(10,0)$ to a constant intermediate state $\left(M^{*}, \theta^{*}\right)$ followed by a 2-rarefaction connecting $\left(M^{*}, \theta^{*}\right)$ to the right state $\left(M_{r}, \theta_{r}\right)=(10,50)$, i.e. $\left(M_{r}, \theta_{r}\right)$ is within zone $\mathrm{N}$ on figure 4. Numerical results are shown on figure 18. The superiority and the necessity of a higher order scheme is clearly stated on this case. 


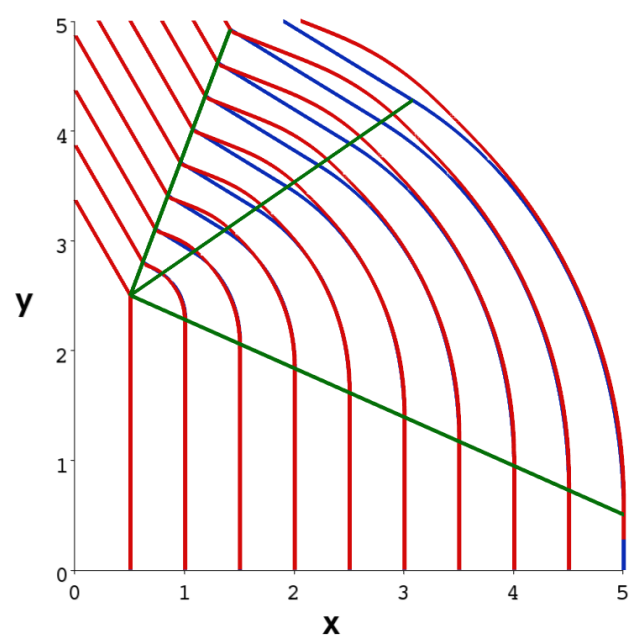

(a) 1st order

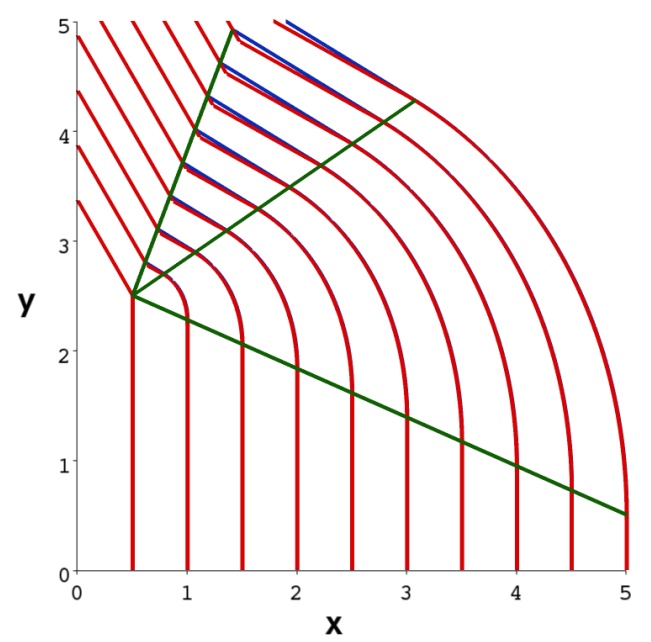

(b) 2nd order

Figure 15: 1-rarefaction + 2-shock: $\left(M_{\ell}, \theta_{\ell}\right)=(10,0)$ and $\left(M_{r}, \theta_{r}\right)=(5,30)$. Same drawing convention.

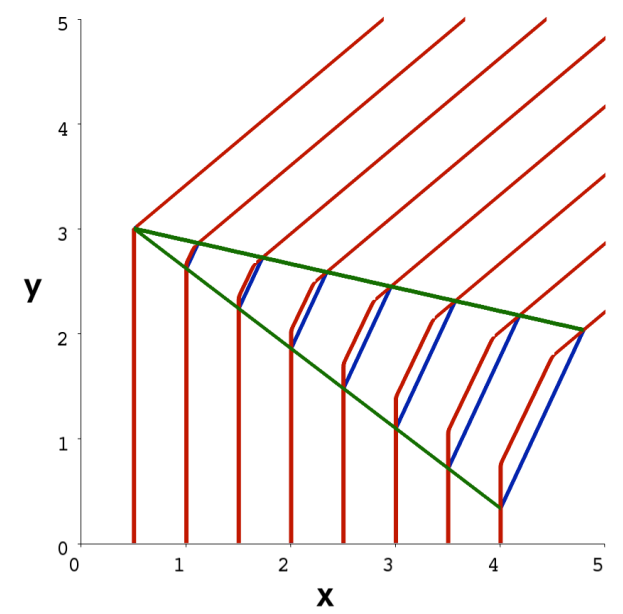

(a) 1st order

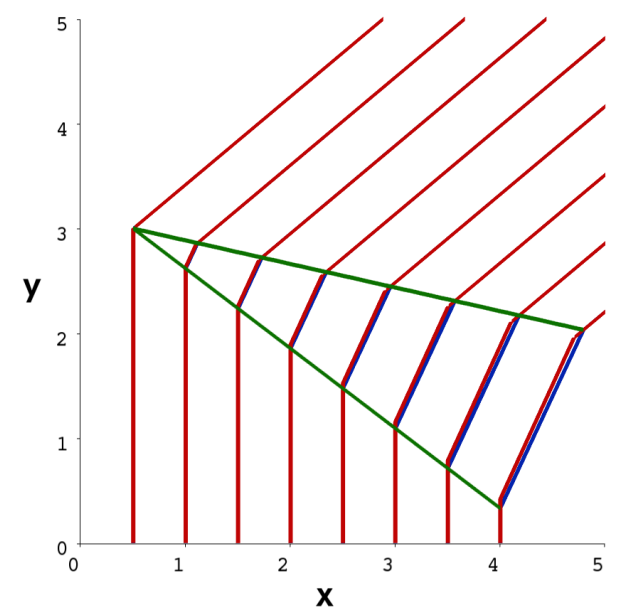

(b) 2nd order

Figure 16: 1-shock +2-shock: $\left(M_{\ell}, \theta_{\ell}\right)=(10,0)$ and $\left(M_{r}, \theta_{r}\right)=(10,-50)$. Same drawing convention. 


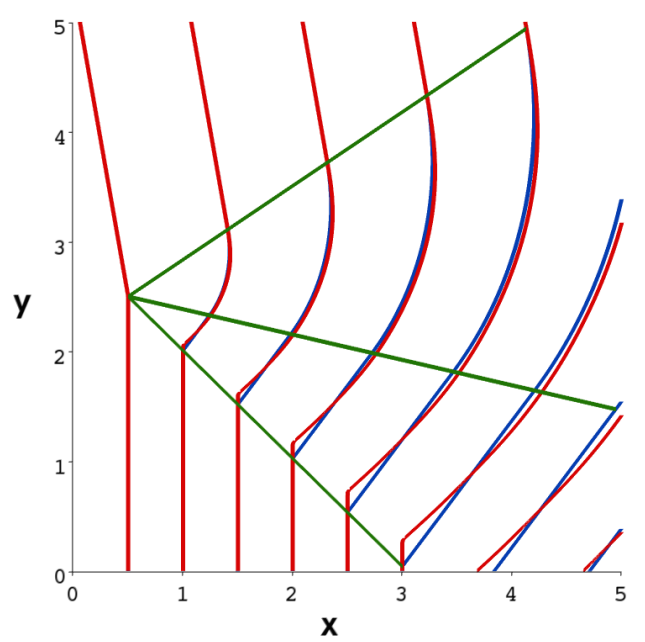

(a) 1st order

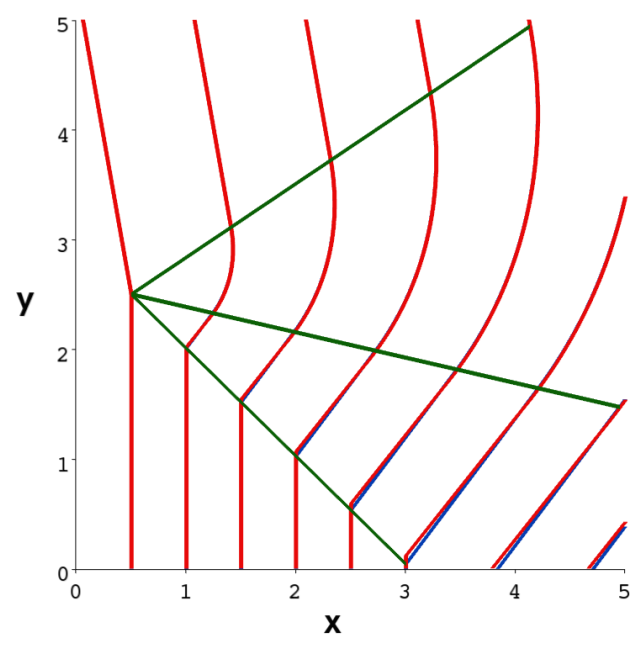

(b) 2nd order

Figure 17: 1-shock + 2-rarefaction: $\left(M_{\ell}, \theta_{\ell}\right)=(10,0)$ and $\left(M_{r}, \theta_{r}\right)=(20,10)$. Same drawing convention.

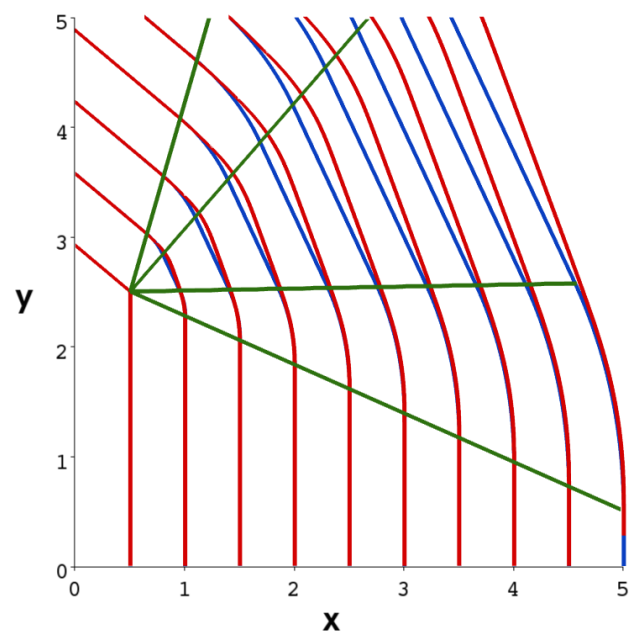

(a) 1st order

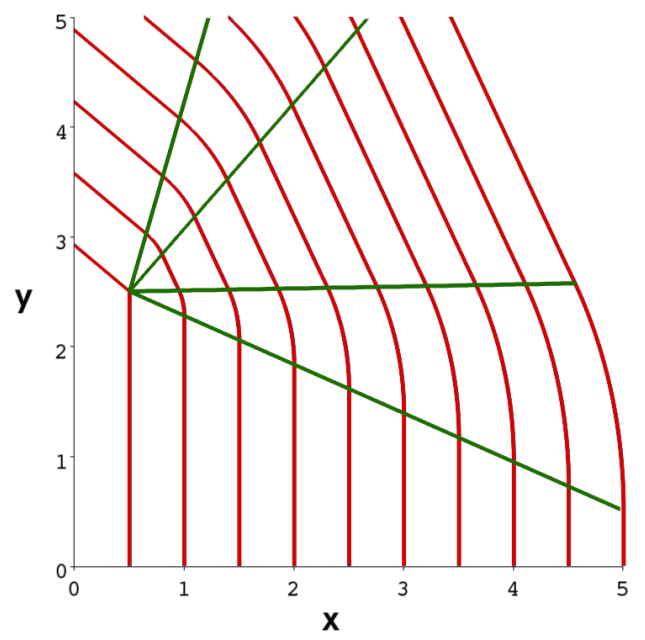

(b) 2 nd order

Figure 18: 1-rarefaction + 2-rarefaction: $\left(M_{\ell}, \theta_{\ell}\right)=(10,0)$ and $\left(M_{r}, \theta_{r}\right)=(10,50)$. Same drawing convention.

\subsubsection{Synthesis}

In view of the results obtained in this series of test cases, we note that both first-order and second-order numerical schemes give, qualitatively, the 
expected behaviour of the exact solution to the Riemann problem. However, there are substantial differences between the results obtained by each order. It is observed that the first-order scheme is not accurate enough, which leads to a significant discrepancy on the position of the shock, while the second-order scheme provides a much better approximation and coincides quite strictly with the exact solution for all cases.

\section{Conclusion}

In this paper, we described a new algorithm for solving the Geometrical Shock Dynamics model. In this hyperbolic two equations model, the arrival time, $\alpha(\mathbf{x})$, of a shock wave is given by a nonlinear eikonal equation whose local speed, or Mach number $M(\mathbf{x})$, is governed by a differential equation depending on flow parameters and the front curvature. By reformulating the transport equation on $M$ as a convection-diffusion one, its approximation is made easier and the lengthy discretization of the mean curvature is avoided. The new algorithm follows the fast-marching method on a cartesian grid but uses trial values when updating the current node. First derivatives, appearing in the eikonal and transport equations, are upwind sided up to second order depending on the causality condition on $\alpha$. A key feature of the method is to use a centered discrete Laplacian for the diffusion part, as much as possible, whithout taking into account the causality condition (i.e. using points which are not in the Known set). For this reason, the algorithm is not a fast-marching method in the strictest sense, and we say it has fastmarching like properties. The resulting algebraic nonlinear system is solved by an iterative procedure, fixed point or Newton's method, at each node.

For validation purpose, reference solutions are built for a smooth radial expansion wave and the interaction of two shocks. This latter case is a Riemann problem for which a link is made with the p-system in two dimensions. A detailed analysis of this problem is provided and simple front characterization is given in the strong shock limit for any left and right states. In general, those states are connected by shock or rarefaction waves developing on the front.

Numerical experiments have shown that, as expected, the algorithm is second order for smooth fronts and performs remarkably well on discontinous solutions, although the non conservative model equations are solved. Differences are however observed between the first and second order algorithm on the Riemann problems. The second order scheme is much better 
and very close to the reference solution, except when it locally switches to first order. This is particularly noticeable on the compression wedge case where the second order scheme fails above a wedge angle of nearly 45 degrees. This is not a severe limitation in pratice since the GSD model is not expected to give accurate results in such a case.

In the future we plan to extend our algorithm to deal with immersed rigid bodies and optimize the solver to achieve very fast computations. At last, we infer that this new algorithm could also be valuable to the detonic community, mainly for Detonation Shock Dynamics [26].

\section{Appendix A. Reference solutions of the Riemann problem in the strong shock limit}

Based on the geometric description of section 3, we outline the construction of explicit solutions, in the strong shock limit $(M>>1)$, for the 2D Riemann problem. For the sake of simplicity, we give the explicit solution for specific configurations only: simple waves and a particular case with an intermediate state. The key tool is to use the simplified relation (29) between the area section and the Mach number.

We consider initial constant left and right states joined at the origin $O=(0,0)$ and use the polar coordinates $x=\rho \cos \chi$ and $y=\rho \sin \chi$.

\section{A.1. Simple shock wave}

From the limit form (29) of the A-M relation in the strong shock limit, the equations (26)-(27) and (25)-(27) become (see also (30))

$$
\left\{\begin{aligned}
\cos \left(\theta_{\ell}-\theta_{r}\right) & =\frac{m^{\lambda_{\infty}}+m}{1+m^{\lambda_{\infty}+1}} \\
\tan \left(\chi_{\nu}^{s}-\theta_{\ell}\right) & =[-1+\nu(\nu-1)] m^{\lambda_{\infty}}\left(\frac{1-m^{2}}{1-m^{2 \lambda_{\infty}}}\right)^{1 / 2}
\end{aligned}\right.
$$

where $m=\frac{M_{r}}{M_{\ell}}$ and $\nu=1,2$ for the 1 -shock or 2-shock cases. Given $0<$ $\theta_{\ell}-\theta_{r}<\frac{\pi}{2}$, from the first equation of this algebraic system, one deduces, by an iterative procedure, the Mach number ratio $m$ on each side of the shockshock singularity. This singularity is characterized by the angle $\chi_{\nu}^{s}$ which is obtained by injecting $m$ in the second equation. 


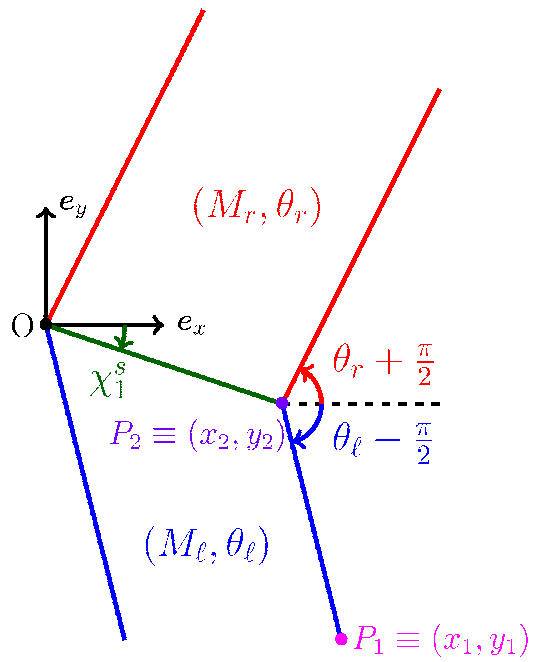

Figure A.19: Example of geometric construction of 1-shock.

For this case, the geometric construction is straightforward and proceeds as follows. As shown in figure A.19, we consider the two angular sectors separated by the shock line extending from the origin $O$ and having the slope $\tan \chi_{\nu}^{s}$. At a given time, one starts from a current position $P_{1} \equiv\left(x_{1}, y_{1}\right)$ on the left state and draws the line segment whose normal makes the angle $\theta_{\ell}$ with the $(O x)$ axis. The other endpoint of this segment, $P_{2} \equiv\left(x_{2}, y_{2}\right)$, lies on the shock line and so has the polar angle $\chi_{\nu}^{s}$. This process is repeated to draw the front shape at different times to cover the computational domain.

The scalar function $\alpha$, that characterizes the successive front positions, is then (see also figure 3 )

$$
\alpha(x, y)=\left\{\begin{array}{ll}
\frac{x \cos \theta_{\ell}+y \sin \theta_{\ell}}{M_{\ell}}, & \chi \leq \chi_{\nu}^{s} \\
\frac{x \cos \theta_{r}+y \sin \theta_{r}}{M_{r}}, & \chi \geq \chi_{\nu}^{s}
\end{array} .\right.
$$

\section{A.2. Simple rarefaction wave}

As mentioned in section 3.2, the rarefaction locus is a spiral

$$
\rho=t_{0} \rho_{1}(\chi) \text { where } t_{0}>0,
$$


connecting the two constant states. In the strong shock limit $(M>>1)$, this spiral is of logarithmic type, and the angle between its normal and the radial direction is constant (see (15)) and is given by

$$
\delta_{\infty}=\arctan \sqrt{\frac{1}{\lambda_{\infty}}} .
$$

In other words, the angles in Proposition 3.1 are written

$$
\chi_{1,2}^{r-}=\theta_{\ell} \mp \delta_{\infty} \quad \text { and } \quad \chi_{1,2}^{r+}=\theta_{r} \mp \delta_{\infty},
$$

where the signs $\mp$ refer to the 1-rarefaction and the 2-rarefaction respectively. Moreover equation (23) simplifies to give

$$
\theta_{r}-\theta_{\ell}=\mp \sqrt{\lambda_{\infty}} \log \left(\frac{M_{r}}{M_{\ell}}\right)
$$

with $\pi>\theta_{r}-\theta_{\ell}>0$ (see Proposition 3.1).

The scalar function $\alpha$, that characterizes the successive positions of the shock front, is then written (see figure 2)

$$
\alpha(x, y)=\left\{\begin{array}{l}
\frac{x \cos \theta_{\ell}+y \sin \theta_{\ell}}{M_{\ell}}, \chi \leq \theta_{\ell} \mp \delta_{\infty} \\
K \rho \mathrm{e}^{ \pm \frac{\chi}{\sqrt{\lambda_{\infty}}}}, \theta_{\ell} \mp \delta_{\infty} \leq \chi \leq \theta_{r} \mp \delta_{\infty} . \\
\frac{x \cos \theta_{r}+y \sin \theta_{r}}{M_{r}}, \chi \geq \theta_{r} \mp \delta_{\infty}
\end{array}\right.
$$

with $K=\frac{\cos \delta_{\infty}}{M_{\ell}} e^{\mp \frac{\chi_{1,2}^{r-}}{\sqrt{\lambda \infty}}}$

\section{A.3. Complete solutions of the Riemann problem}

Whatever the left and right initial conditions, the intermediate state $\left(\theta^{*}, M^{*}\right)$ can be written in the generic form:

$$
\left\{\begin{array}{rl}
\theta^{*}-\theta_{\ell} & =\mathcal{G}_{\mathrm{R}, \mathrm{S}}\left(\frac{M^{*}}{M_{\ell}}\right) \\
\theta_{r}-\theta^{*} & =\mathcal{G}_{\mathrm{R}, \mathrm{S}}\left(\frac{M^{*}}{M_{r}}\right)
\end{array},\right.
$$


where, in the strong shock limit, the function $\mathcal{G}_{\mathrm{R}, \mathrm{S}}$ is given by

$$
\mathcal{G}_{\mathrm{R}, \mathrm{S}}(m)=\mid \begin{array}{ll}
\mathcal{G}_{\mathrm{R}}(m)=-\sqrt{\lambda_{\infty}} \log m & \text { for a rarefaction } \\
\mathcal{G}_{\mathrm{S}}(m)=-\arccos \left(\frac{m+m^{\lambda_{\infty}}}{1+m^{1+\lambda_{\infty}}}\right) & \text { for a shock }
\end{array}
$$

For illustration purpose, we consider only the 1-rarefaction followed by 2-rarefaction case, for which system (A.1) can be solved explicitly. One then checks that

$$
\left\{\begin{array}{rl}
\theta^{*} & =\frac{\theta_{r}+\theta_{\ell}-\sqrt{\lambda_{\infty}} \log \left(\frac{M_{r}}{M_{\ell}}\right)}{2} \\
M^{*} & =\sqrt{M_{r} M_{\ell} e^{\frac{\theta_{\ell}-\theta_{r}}{\sqrt{\lambda_{\infty}}}}}
\end{array} .\right.
$$

Remark A.1. For any other configuration, an iterative procedure is required to determine the intermediate state.

For the example under consideration, the front is compound of five angular sectors, according to the values of the polar angle $\chi$ (see figure 5). The Riemann solution consists in two spiral arcs, characterized respectively by the angles $\pm \delta_{\infty}$, connecting the intermediate state to the plane shocks on the left and on the right respectively. Note that in the region between the two spiral arcs, the shock is plane (i.e. $M=M^{*}$ and $\theta=\theta^{*}$ ) and tangent to both curves. More precisely, the function $\alpha$ is written

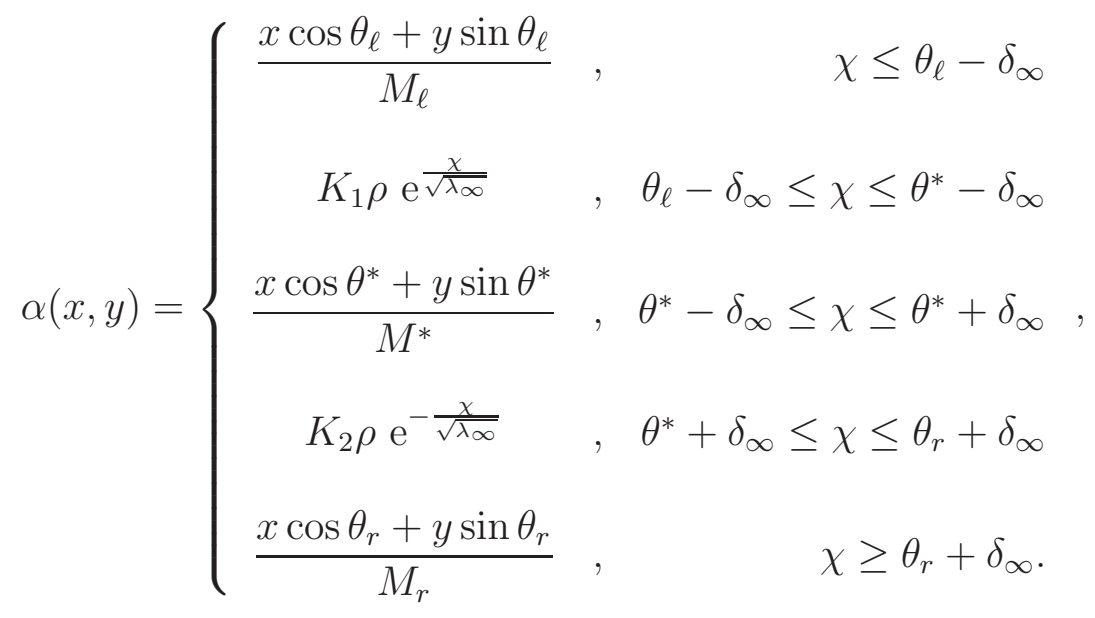


with

$$
K_{1}=\frac{\cos \delta_{\infty}}{M_{\ell}} e^{-\frac{\left(\theta_{\ell}-\delta_{\infty}\right)}{\sqrt{\lambda_{\infty}}}}, \quad K_{2}=\frac{\cos \delta_{\infty}}{M_{r}} e^{\frac{\left(\theta_{\ell}+\delta_{\infty}\right)}{\sqrt{\lambda_{\infty}}}},
$$

such that the function $\alpha$ is obviously continuous globally.

\section{References}

[1] Anand, R.K., "Shock dynamics of strong imploding cylindrical and spherical shock waves with non-ideal gas effects", Wave Motion, vol. 50, pp. 1003-1015, 2013.

[2] Aslam, T.D., "Investigations on Detonation Shock Dynamics", PhD Thesis, University of Illinois at Urbana-Champaign, U.S.A., 1996.

[3] Aslam, T.D., Bdzil, J.B., Stewart, D.S., "Level set methods applied to modeling detonation shock dynamics", Journal of Computational Physics, vol. 126, pp. 390-409, 1996.

[4] Aslam, T.D., Stewart, D.S., "Detonation shock dynamics and comparison with direct numerical simulation", Combustion Theory Modelling, 1999.

[5] Baskar, S., Prasad, P., "Propagation of curved shock fronts using shock ray theory and comparison with other theories", J. Fluid Mech., vol. 5, pp. 171-198, 2005.

[6] Bdzil, J.B., Aslam, T.D., "Detonation front models: Theories and methods", LA-UR 00-942, 1999.

[7] Besset, C., Blanc, E., "Propagation of vertical shock waves in the atmosphere", J. Acoust. Soc. Am., vol. 95(4), pp. 1830-1839, 1994.

[8] Best, J.P., "A generalisation of the theory of geometrical shock dynamics", Shock Waves, vol. 1(4), pp. 251-2739, 1991.

[9] Best, J.P., Erratum: "A generalisation of the theory of geometrical shock dynamics", Shock Waves, vol. 2, pp. 125, 1992.

[10] Best, J.P., "Accounting for Transverse Flow in the Theory of Geometrical Shock Dynamics", Proc. R. Soc. A., vol. 442 (no. 1916), pp. 585-598, 1993. 
[11] Cates, J.E., Sturtevant, B., "Shock wave focusing using geometrical shock dynamics", Physics of Fluids, vol. 9(10), pp. 3058-3068, 1997.

[12] Crandall, M.G., Lions, P.L., "Two approximation solutions of HamiltonJacobi equations", Math. Comp., 43, 1-19, 1984.

[13] Dafermos, C.M., "Hyperbolic Conservation Laws in Continuum Physics", Grundlehren der mathematischen Wissenschaften, vol. 325, 3rd ed., Heidelberg, New York, Springer, (2010).

[14] Goodman, J., MacFayden, A., "Ultra-relativistic geometrical shock dynamics and vorticity", Journal of Fluid Mechanics, vol. 604, pp. 325-338, 2008.

[15] Henshaw, W.D., Smyth, N.F., Schwendeman, D.W., "Numerical shock propagation using geometrical shock dynamics", Journal of Fluid Mechanics, vol. 171, pp. 519-545, 1986.

[16] Osher, S., Fedkiw, R., "Level Set Methods and Dynamic Implicit Surfaces", volume 153 of Applied Mathematical Sciences, Springer, 2003.

[17] Osher, S., Sethian, J.A., "Fronts Propagating with CurvatureDependent Speed: Algorithms Based on Hamilton-Jacobi Formulations", Journal of Computational Physics, vol. 79, pp. 12-49, 1988.

[18] Schwendeman, D.W., "A numerical scheme for shock capturing in three dimensions", Proc. Royal Society. Lond., A416 (no. 1850), pp. 179-198, 1988.

[19] Schwendeman, D.W., "A new numerical method for shock wave propagation using geometrical shock dynamics", Proc. Royal Society. Lond., A441 (no.1912), pp. 331-341, 1993.

[20] Schwendeman, D.W., "A higher order Godunov method for the hyperbolic equations modeling shock dynamics", Proc. Royal Society Lond., A455 (no. 1984), pp. 1215-1233, 1999.

[21] Serre, D., "Systems of conservation laws. 1. Hyperbolicity, entropies, shock waves". Translated from the 1996 French original by I. N. Sneddon. Cambridge University Press, Cambridge, 1999 
[22] Sethian, J.A., "Level Set Methods and Fast Marching Methods: Evolving Interfaces in Computational Geometry, Fluid Mechanics, Computer Vision and Materials Science", Cambridge University Press, 1999.

[23] Sethian, J.A., Smereka, P., "Level set methods for fluid interface", Annu. Rev. Fluid Mech., vol. 35, pp. 341-372, 2003.

[24] Smoller, J., "Shock Waves and Reaction-Diffusion Equations", Grundlehren der Mathematischen Wissenschaften [Fundamental Principles of Mathematical Sciences], 258, Springer-Verlag, New York, 1994.

[25] Shu, C.W., Osher, S., "High-order essentially non oscillatory schemes for Hamilton-Jacobi equations", SIAM J. Numer. Anal, vol. 28(4), pp. 907-922, 1991.

[26] Stewart, D.S., Bdzil, J.B., "The shock dynamics of stable multidimensional detonation", Combustion and Flame, vol. 72, pp. 311-323, 1988.

[27] Varadarajan, P.A., Roe, P.L., "Geometrical shock dynamics and engine unstart", 41st AIAA Fluid Dynamics Conference and Exhibit, 27-30 June 2011, Honolulu, Hawaii.

[28] Whitham, G.B., "A new approach to problems of shock dynamics. Part I, Two dimensional problems", J. Fluid. Mech., vol. 2, pp. 146-171, 1957.

[29] Whitham, G.B., "Linear and Nonlinear Waves", Wiley (New York), 1974.

[30] Yatziv, L., Bartesaghi, A., Sapiro, G., "A Fast O(N) Implementation of the Fast Marching Algorithm", Journal of Computational Physics, vol. 212, pp. 393-399, 2006.

[31] Zakeri, G.A., "Analysis of ray tube area in geometrical shock dynamics", Applied Mathematics and Computation, vol. 217(2), pp. 830-836, 2010. 